\title{
Endothelial HIF-2 mediates protection and recovery from ischemic kidney injury
}

\author{
Pinelopi P. Kapitsinou,,1,2 Hideto Sano,,1,2 Mark Michael,,1,2 Hanako Kobayashi,1,2 Olena Davidoff,,1,2 \\ Aihua Bian, ${ }^{3}$ Bing Yao, ${ }^{1}$ Ming-Zhi Zhang, ${ }^{1}$ Raymond C. Harris, ${ }^{1}$ Kevin J. Duffy, ${ }^{4}$ \\ Connie L. Erickson-Miller, ${ }^{5}$ Timothy A. Sutton, ${ }^{6}$ and Volker H. Haase ${ }^{1,2}$

\begin{abstract}
1Department of Medicine, ${ }^{2}$ Departments of Cancer Biology and Molecular Physiology and Biophysics, and ${ }^{3}$ Department of Biostatistics, Vanderbilt University School of Medicine, Nashville, Tennessee, USA. ${ }^{4}$ Cancer Research and ${ }^{5}$ Haemoglobin DPU, GlaxoSmithKline, Collegeville, Pennsylvania, USA. ${ }^{6}$ Department of Medicine, Indiana University School of Medicine, Indianapolis, Indiana, USA.
\end{abstract}

\begin{abstract}
The hypoxia-inducible transcription factors HIF-1 and HIF-2 mediate key cellular adaptions to hypoxia and contribute to renal homeostasis and pathophysiology; however, little is known about the cell type-specific functions of HIF-1 and HIF-2 in response to ischemic kidney injury. Here, we used a genetic approach to specifically dissect the roles of endothelial HIF-1 and HIF-2 in murine models of hypoxic kidney injury induced by ischemia reperfusion or ureteral obstruction. In both models, inactivation of endothelial HIF increased injury-associated renal inflammation and fibrosis. Specifically, inactivation of endothelial HIF-2 $\alpha$, but not endothelial HIF-1 $\alpha$, resulted in increased expression of renal injury markers and inflammatory cell infiltration in the postischemic kidney, which was reversed by blockade of vascular cell adhesion molecule-1 (VCAM1) and very late antigen-4 (VLA4) using monoclonal antibodies. In contrast, pharmacologic or genetic activation of HIF via HIF prolyl-hydroxylase inhibition protected wild-type animals from ischemic kidney injury and inflammation; however, these same protective effects were not observed in HIF prolyl-hydroxylase inhibitortreated animals lacking endothelial HIF-2. Taken together, our data indicate that endothelial HIF-2 protects from hypoxia-induced renal damage and represents a potential therapeutic target for renoprotection and prevention of fibrosis following acute ischemic injury.
\end{abstract}

\section{Introduction}

ECs play a critical role in the pathophysiology of acute and chronic ischemic injuries, as they are involved in the regulation of vascular tone, trafficking of inflammatory cells, delivery of nutrients and oxygen to surrounding cells, wound healing, and tissue repair (1). Because of its highly specialized vascular anatomy and the relatively low tissue $\mathrm{pO}_{2}$ levels, the kidney is particularly susceptible to hypoxic injury, which in hospitalized patients frequently results in acute ischemic renal failure, a condition associated with high mortality and transition to chronic kidney disease (CKD) $(2,3)$. In the hypoxic and/or ischemic kidney, endothelial damage leads to multiple pathologic changes, which include increased vascular permeability, enhanced endothelium-leukocyte interaction with concomitant capillary obstruction and inflammatory cell infiltration, abnormal coagulation, vasoconstriction, and altered vascular growth factor regulation $(4,5)$.

Like other cell types, ECs respond to changes in tissue $\mathrm{PO}_{2}$ levels by multiple hypoxic signaling mechanisms. Central mediators of cellular adaptation to $\mathrm{O}_{2}$ deprivation are hypoxia-inducible transcription factors HIF-1 and HIF-2, pleiotropic heterodimeric basic helix-loop-helix transcription factors that regulate cellular energy metabolism, angiogenesis, erythropoiesis, apoptosis, and cell proliferation (6). The activity of HIFs is controlled by $\mathrm{O}_{2}$, , iron- and ascorbate-dependent dioxygenases, also known as prolyl-4-hydroxylase domain-containing proteins 1-3 (PHD1-3), which use

Conflict of interest: Volker H. Haase serves on the Scientific Advisory Board of Akebia Therapeutics, a company that develops prolyl-4-hydroxylase inhibitors for the treatment of anemia. Connie L. Erickson-Miller and Kevin J. Duffy are employees of GlaxoSmithKline and own stocks.

Citation for this article: J Clin Invest. 2014;124(6):2396-2409. doi:10.1172/JCI69073. 2-oxoglutarate (2OG) as substrate for the hydroxylation of specific proline residues within the oxygen-sensitive HIF- $\alpha$ subunit. This permits binding to the PVHL-E3 ubiquitin ligase complex and results in proteasomal degradation of HIF- $\alpha$ under normoxia (7).

In the noninjured kidney, HIF- $1 \alpha$ has been detected in tubular epithelium and in ECs following exposure to acute hypoxia, while HIF- $2 \alpha$ is predominantly expressed in ECs and glomerular cells as well as in peritubular interstitial cells, where it regulates erythropoietin (EPO) synthesis $(8,9)$. Increased expression of HIF-1 $\alpha$ and HIF- $2 \alpha$ has been found in both acute and chronic kidney injury; however, the role of HIF-1 and HIF-2 in the pathogenesis of renal diseases is not clear (9). In particular, little is known about cell type-specific functions of individual HIF homologs in the context of hypoxic kidney injury (HIF-1 versus HIF-2). HIF has been shown to promote tolerance to acute ischemia, as systemic HIF activation protects from ischemia-reperfusion injury (IRI) in animal models of acute renal failure (10-12). This protective role of HIF in kidney injury appears to be dependent on the timing of its activation $(12,13)$

To understand the effects of endothelial HIF signaling on hypoxic kidney injury and to specifically dissect the individual roles of EC-derived HIF-1 and HIF-2 in renal repair, we used a genetic approach to activate or ablate both HIF homologs either simultaneously or individually by Cre-loxP-mediated recombination. Here, we show that endothelial HIF protects from renal injury and inflammation induced by either renal ischemia-reperfusion or unilateral ureteral obstruction (UUO). We furthermore demonstrate that endothelial HIF-1 and HIF-2 play distinct roles in the pathogenesis of renal IRI, as HIF-2 and not HIF-1 suppressed IRI-associated inflammation and injury and mediated the renoprotective effects of systemic HIF prolyl-hydroxylase inhibition. 
A

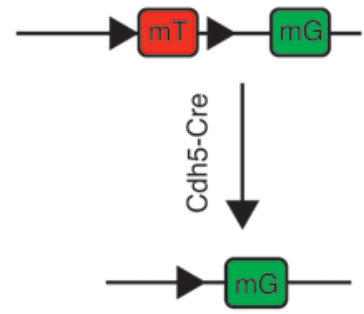

B

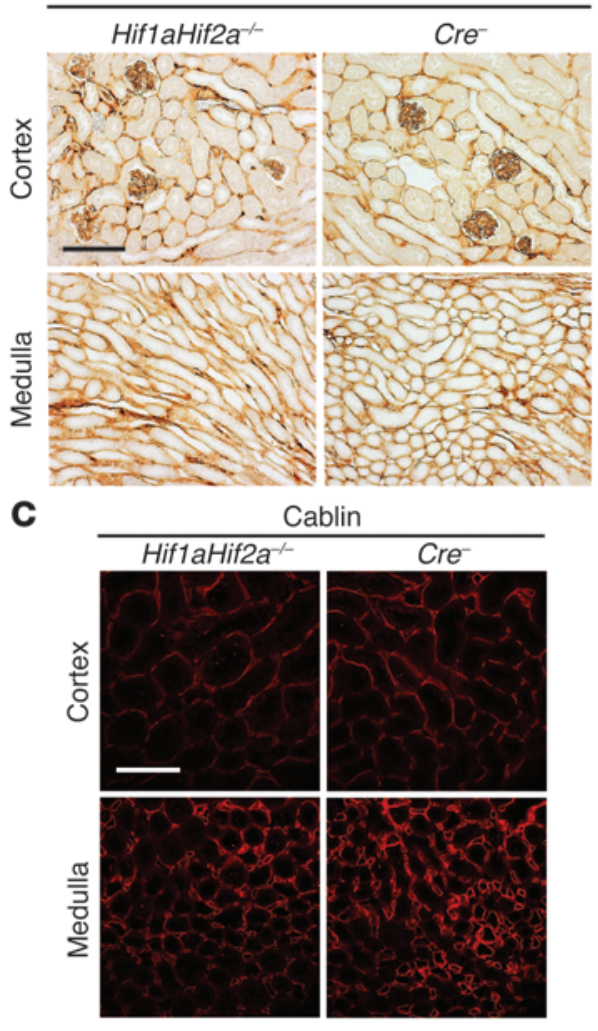

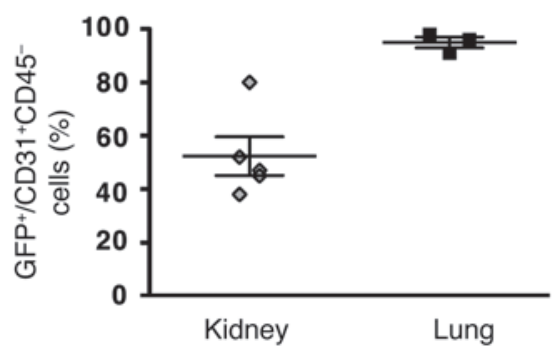

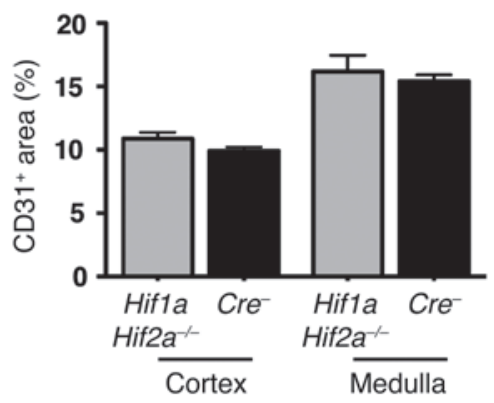

D

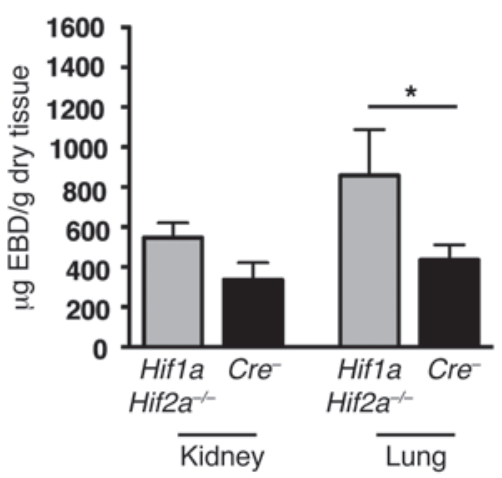

Figure 1

Characterization of mice with ECspecific inactivation of HIF- $1 \alpha$ and HIF-2 $\alpha$. (A) Scheme illustrating the strategy used to assess the degree of EC-specific recombination. Cdh5-Cre mice were crossed to ROSA26-ACTBtdTomato,-EGFP reporter ( $m T / m G$ ) mice, and ECs were analyzed by FACS. Graph on the right shows the percentage of GFP-positive cells contained within the CD31-positive/ CD45-negative cell population isolated from kidneys or lungs. (B) Left: representative images of CD31-stained cortex and medulla of kidneys from EC-specific Hif1aHif2a ${ }^{-/}$and $\mathrm{Cre}^{-}$ control mice. Right: quantification of CD31-positive area $(n=5)$. (C) Shown are representative images of cablinstained peritubular capillaries in renal cortex and medulla imaged with confocal laser-scanning microscopy. The anti-cablin antibody used here preferentially stains the basal lamina of peritubular renal capillaries and does not label glomerular capillaries or small arterioles. (D) Quantification of baseline vascular permeability in kidney and lung tissue from 8-weekold mice using the EBD vascular permeability assay $(n=4-6)$. Graph bars represent mean values \pm SEM; ${ }^{\star} P<0.05$. Scale bars: $100 \mu \mathrm{m}$.
Taken together, our studies identify endothelial HIF-2 as a critical therapeutic target that can be activated pharmacologically to induce renoprotection.

\section{Results}

Generation and phenotypic characterization of mice with EC-specific inactivation of HIF-1 $\alpha$ and HIF-2 $\alpha$. To investigate the role of endothelial HIF in the context of kidney injury, we crossed the VE-cadherin-Cre (Cdh5-Cre) transgene (14) to conditional Hif1a and Hif2a (Epas1) alleles $(15,16)$ and generated mice that lacked both HIF- $\alpha$ homo-

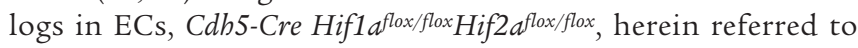
HiflaHif2 $a^{-/-}$mutants. Genomic PCR analysis was used to assess recombination in adult lungs, heart, liver, and kidneys (Supplemental Figure 1A; supplemental material available online with this article; doi:10.1172/JCI69073DS1). To further characterize the degree of EC recombination, Cdh5-Cre mice were intercrossed with double-fluorescent Cre-reporter mice, which expressed red fluorescent membrane-bound tdTomato prior to excision and GFP membrane-bound EGFP following excision of a floxed stop cassette (ref. 17 and Figure 1A). CD31-positive/CD45-negative single cell suspensions isolated from lung and kidney tissue were analyzed for EGFP expression by FACS. Compared with lungs, where $95 \% \pm 2 \%$ of ECs stained positive for EGFP, Cre-recombinase was active in $52 \% \pm 7 \%$ of ECs isolated from renal tissue $(n=3-5$; Figure 1A). EGFP was uniformly distributed in both glomerular ECs and peritubular capillaries of the renal interstitium (Supplemental Figure 1B), and no preferential expression of endothelial markers CD31 and CD34 cells was detected in EGFP-positive and EGFP-negative ECs by FACS analysis (data not shown).

HiflaHif2 $a^{-/-}$mice were born at the expected Mendelian frequency and did not display any apparent pathology (Supplemental Figure 1C). A defect in erythropoiesis, which had been previously reported in hypomorph Hif2a knockdown mice (18), was not observed in Hif1aHif2a $a^{-/-}$mutants (Supplemental Table 1). Furthermore, measurements of renal function parameters, such as blood urea nitrogen (BUN), serum electrolytes, urine albumin, and Epo levels, revealed

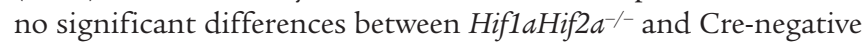
Hif1 $a^{\text {flox/flox }} \mathrm{Hif} 2$ a flox/flox mice, herein referred to as $\mathrm{Cre}^{-}$littermate controls (Supplemental Table 1). These results are consistent with normal morphology of Hif1aHif2a-/- kidneys stained with H\&E (Sup- 
A

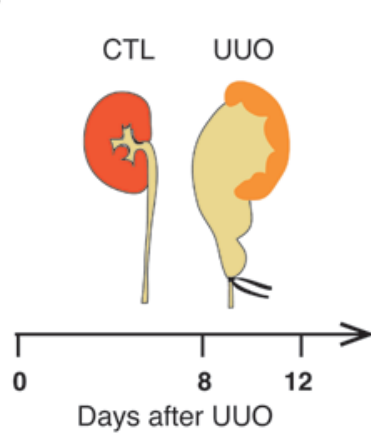

B

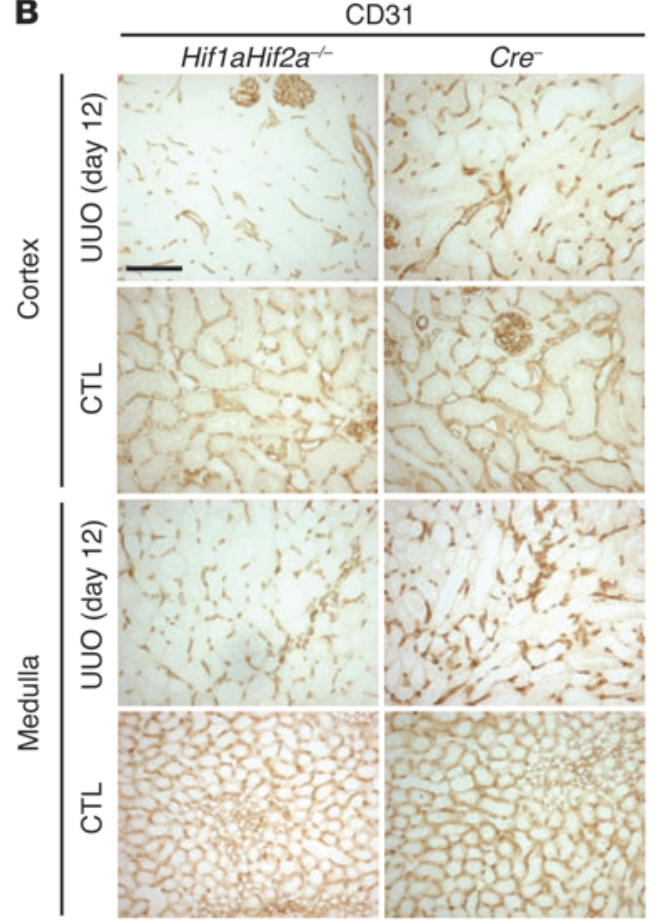

Sirius red

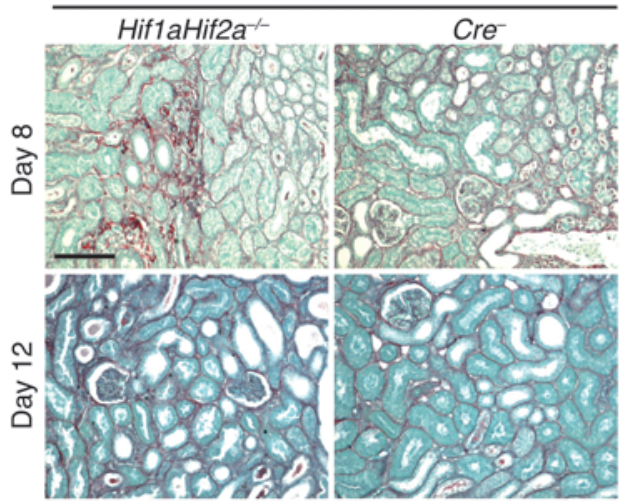

Cortex
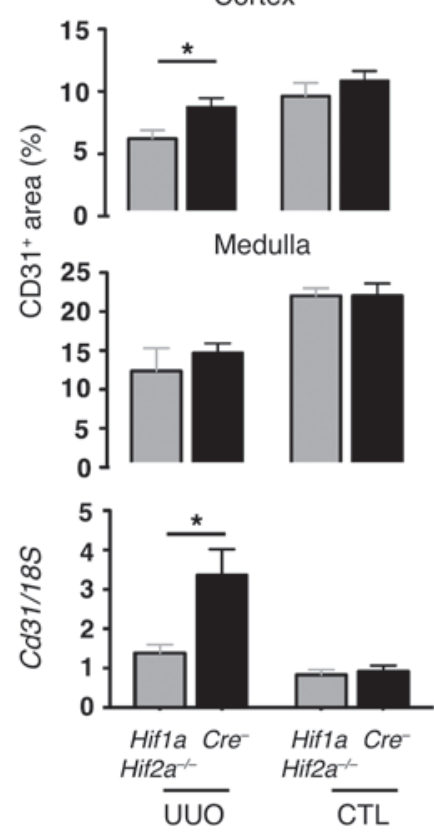

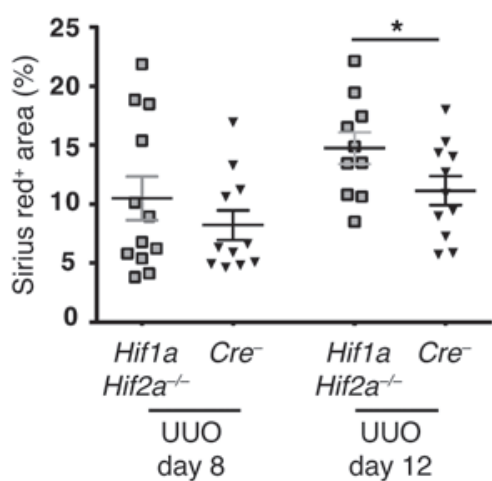

C
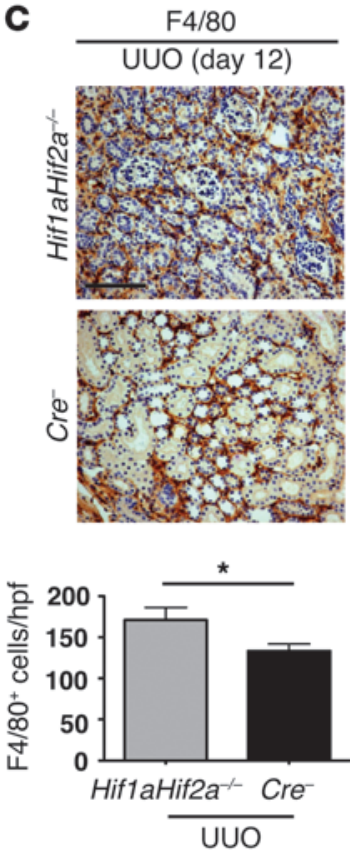

Figure 2

Endothelial HIF protects from UUO-induced renal injury and inflammation. (A) Shown are representative Sirius red-stained kidney sections and corresponding quantification of Sirius red-positive areas at days 8 and 12 following UUO $(n=10-12)$. (B) Representative images of CD31-stained renal cortex or medulla from mutant and control kidneys (day 12 after UUO); panels on right show quantification of CD31-positive areas in cortex or medulla $(n=10)$ and corresponding Cd31 mRNA levels in UUO (day 12) and CTL kidneys from Hif1aHif2a ${ }^{-/-}$mice and controls. (C) Shown are F4/80-stained representative kidney sections from Hif1aHif2a-l- mutants and Cre- mice at day 12 following UUO $(n=10-11)$. Graph shows the number of F4/80-positive cells per hpf. Graph bars represent mean values $\pm \mathrm{SEM}$; ${ }^{*} P<0.05$. UUO, kidney subjected to UUO; CTL, contralateral kidney. Scale bars: $100 \mu \mathrm{m}$.

plemental Figure 1C). We next performed immunohistochemical analysis of CD31 and cablin expression to examine the peritubular

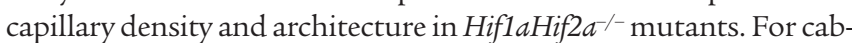
lin immunohistochemistry, we used an antibody that preferentially stains the basal lamina of peritubular renal capillaries (19). Using light and confocal laser scanning microscopy of CD31- and cablinstained kidney sections, we did not detect significant differences in peritubular capillary density or morphology between HiflaHif2a-1mice and $\mathrm{Cre}^{-}$littermates (Figure 1, B and C). We next investigated whether HIF inactivation in ECs affected vascular permeability. Increased extravasation of Evans blue dye (EBD) was observed in the lungs from Hif1aHif2 $a^{-/-}$mice $(858 \pm 231 \mu \mathrm{g} / \mathrm{g}$ tissue vs. $435 \pm 75 \mu \mathrm{g} / \mathrm{g}$ tissue in control littermates, $n=4-6, P=0.04$; Figure 1D), which is in agreement with a prior study that reported enhanced vascular permeability in lungs of mice lacking endothelial HIF-2 $\alpha$ (20). However, vascular permeability in mutant kidneys was not significantly different from that of controls using this assay (Figure 1D), which could have resulted from differences in recombination efficiency or organ-specific HIF functions.

Endothelial HIF protects from UUO-induced renal injury and inflammation. The cell type-specific functions of HIF in hypoxic kidney injury are not well understood. To gain initial insights into 
A

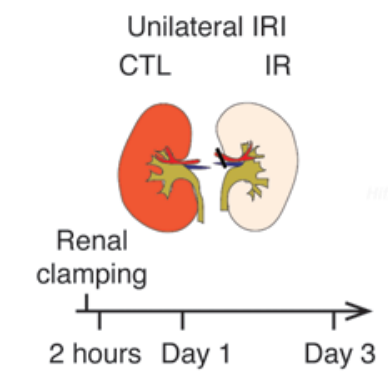

2 hours $p$ IR

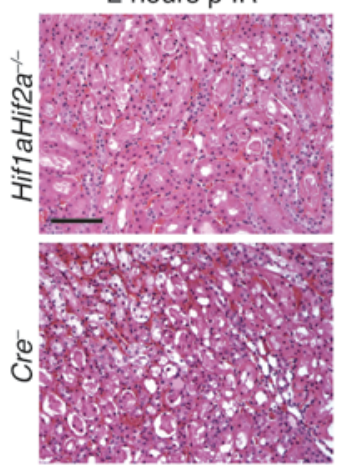

Day 3
Day $1 \mathrm{p} \mathrm{IR}$

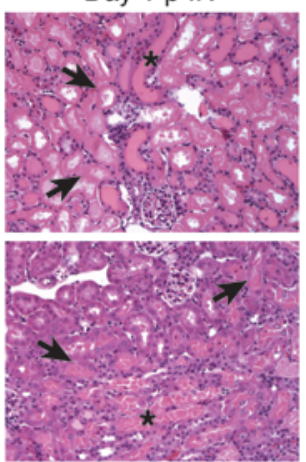

Day $3 \mathrm{p} \mathrm{IR}$

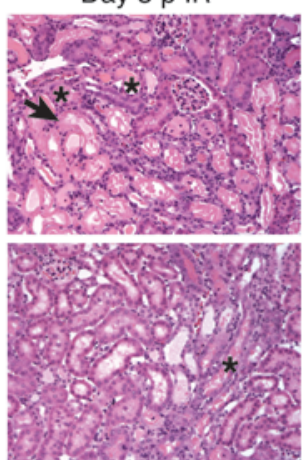

C

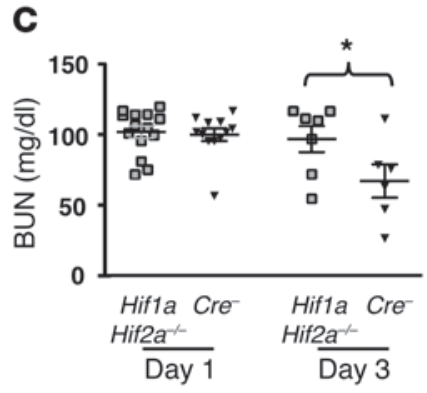

\section{Figure 3}

Endothelial HIF promotes recovery from renal IRI. (A) Schematic illustration of injury model and representative images of H\&E-stained sections of injured kidneys from Hif1aHif2a $a^{-/}$mutants and Cre- littermate controls at 2 hours, 1 day, and 3 days following unilateral IRI. Arrows point to necrotic tubules; asterisks indicate tubules with cast-forming material. (B) Left panel: time course analysis of Kim1 mRNA levels in injured kidneys. Right panel: mRNA levels of Kim1 in injured and contralateral kidneys at day 3 after IRI $(n=5-6)$. (C) BUN levels at day 1 and 3 following bilateral renal IRI in Hif1aHif2a ${ }^{-/-}$and controls $(n=6-14)$. Graph bars represent mean values \pm SEM; ${ }^{*} P<0.05$. IR, kidney subjected to unilateral renal ischemia-reperfusion. Scale bars: $100 \mu \mathrm{m}$.

the role of endothelial HIF in this context, we chose the UUO model, a simple and nonreversible model of rapidly progressing fibrosis and capillary injury, in which kidneys are characterized by hypoxia and HIF activation (21). We first examined renal injury responses in HiflaHif2 $a^{-/-}$mice subjected to UUO at day 8 after ligation, a time point characterized by marked capillary rarefaction, interstitial fibroblast and ECM expansion, increased mononuclear cell infiltration, tubular atrophy, and apoptosis (21). Quantitative analysis of ECM collagen accumulation by Sirius red morphometry revealed increased collagen content in HiflaHif $2 a^{-/-}$mice compared with controls (increase by $28 \%$, $n=11-12, P=0.39$; Figure $2 \mathrm{~A}$ ). By day 12 , this difference became statistically significant and amounted to an approximately $32 \%$ increase $(n=10-11, P=0.032$; Figure 2A). Capillary density as assessed by CD31 immunohistochemistry was reduced by $25 \%$ in the cortex of mutant kidneys ( $n=4-6, P=0.03$; Figure $2 \mathrm{~B}$ ), while significant differences were not detected in the renal medulla. Furthermore, the reduction in cortical capillary density was reflected in a 2.4-fold decrease in Cd31 mRNA isolated from total kidney homogenates $(n=4-6, P=0.009$; Figure $2 B)$. Since ECs play an important role in the regulation of inflammation, which is a characteristic feature of tubulointerstitial kidney injury, we next assessed macrophage numbers by immunohistochemistry. The number of F4/80-positive cells was increased by approximately $25 \%$ in mutant UUO kidneys (171 cells/high-power field [hpf] \pm 15 vs. 131 cells $/ \mathrm{hpf} \pm 8$ in controls, $n=10-11, P=0.038$; Figure $2 \mathrm{C}$ ).
To further investigate whether EC-derived HIF regulates macrophage polarization in this model, we analyzed the expression of M1 and M2 polarization markers in CD11b-positive cells isolated from HiflaHif2 $\mathrm{a}^{-/-}$or $\mathrm{Cr} \mathrm{e}^{-}$UUO kidneys by magnetic bead cell separation. We did not find clear evidence of differences in macrophage polarization, as transcript levels of genes associated with M1 (Il1b, Tnfa, and iNOs) or M2 polarization (Il10, mannose receptor $[M r]$ and arginase $1[\operatorname{Arg} 1]$ ) did not differ between the 2 genotypes (Supplemental Figure 2). Taken together, our data demonstrate that inactivation of HIF in ECs exacerbated UUOinduced fibrosis and led to increased capillary rarefaction and macrophage accumulation, but not to alterations in macrophage $\mathrm{M} 1 / \mathrm{M} 2$ polarization.

Endothelial HIF promotes recovery from renal IRI and inhibits IRI-associated fibrosis. Our findings in a nonreversible renal injury model suggested that endothelial HIF plays a critical role in the regulation of inflammation and capillary maintenance. We therefore hypothesized that HIF signaling in ECs may be important for tissue repair under conditions of reversible hypoxic renal injury. To test this hypothesis, we used a model of acute IRI induced by renal pedicle clamping. HiflaHif2 $a^{-/-}$mutants were subjected to unilateral renal pedicle occlusion for 25 minutes and analyzed at multiple time points following reperfusion. At 2 hours and on day 1 after IRI, endothelial HiflaHif2 $\mathrm{a}^{-/-}$mutants displayed similar degrees of injury compared with Cre- littermate controls, as assessed by morphologic analysis and kidney injury molecule 1 (Kim1) expression 
A

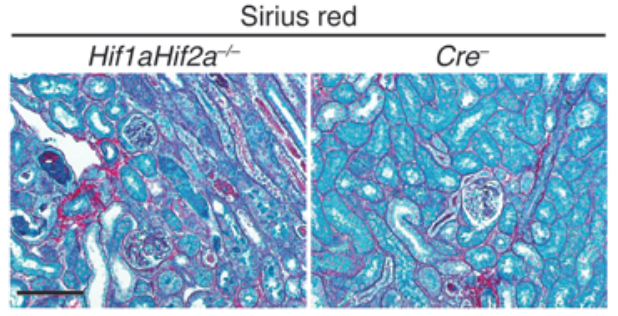

B

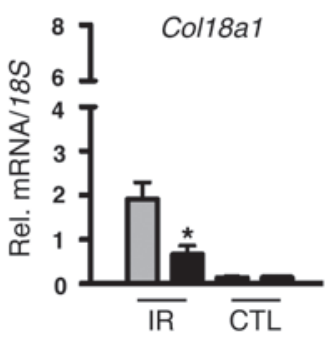

C

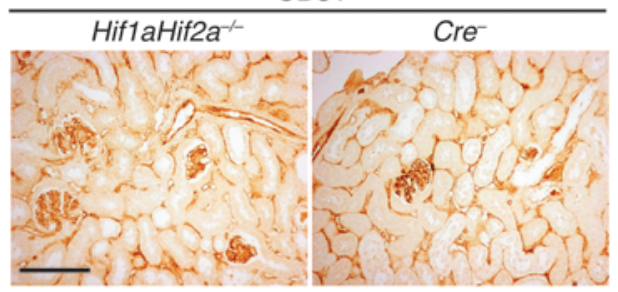

D

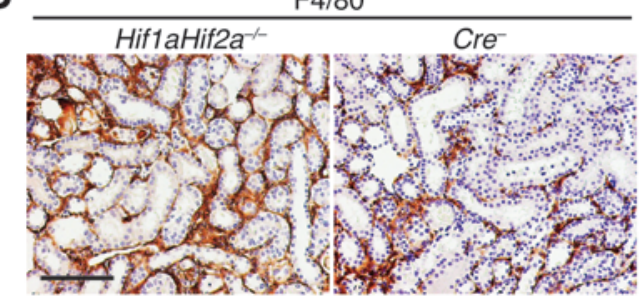

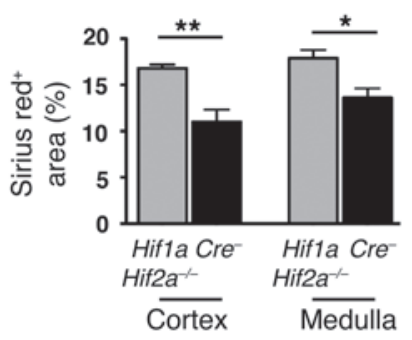

\section{LoxL2}
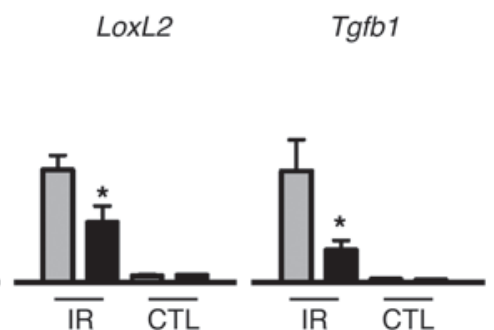

页
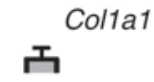
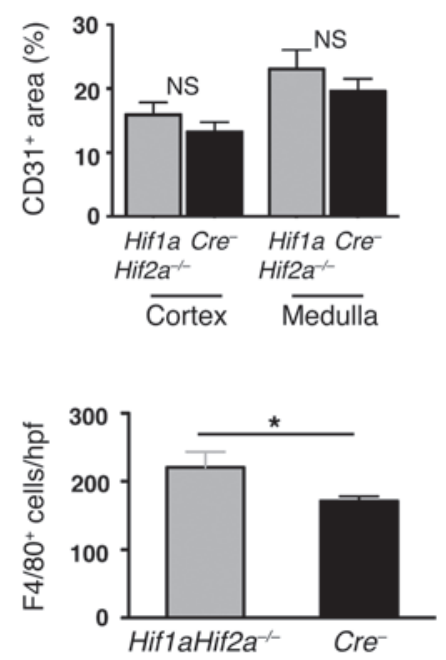

\section{Figure 4}

Inactivation of endothelial HIF promotes IRI-associated tubulointerstitial fibrosis. (A) Representative images of Sirius redstained kidneys from EC-specific Hif1aHif2a-/- and $\mathrm{Cre}^{-}$control mice at day 9 after IRI. Right panel shows quantification of Sirius red-positive area in cortex and medulla $(n=4)$. (B) RTPCR analysis of Col18a1, Loxl-2, Tgfb1, and Col1a1 mRNA levels in mutant and control kidneys at day 9 following IRI $(n=4)$. (C and D) Representative images of CD31- and F4/80-stained kidneys analyzed on day 9 after renal clamping. Right panels: quantification of CD31-positive area and F4/80-positive cell number/hpf $(n=4-5)$. Graph bars represent mean values \pm SEM; ${ }^{*} P<0.05$; ${ }^{\star *} P<0.01$. Scale bars: $100 \mu \mathrm{m}$. levels ( $n=5-6$; Figure 3, A and B). The expression of Kim1, which encodes a transmembrane tubular protein, correlates positively with the degree of renal damage in both acute and chronic injuries (22). On day 3 after IRI, differences in Kim 1 levels became apparent between mutant and control kidneys (1.6-fold increase in mutants, $n=5, P=0.038$; Figure 3B), while overall Kim 1 expression levels were reduced in both genotypes compared with day 1 after IRI. These molecular differences were also reflected in significantly different morphologic injury scores (Supplemental Figure 3).

To investigate whether changes in morphologic injury score and Kim 1 mRNA levels correlated with renal function, HiflaHif $2 a^{-/-}$mice and $\mathrm{Cre}^{-}$littermates were subjected to bilateral renal artery occlusion. To produce sufficient renal injury, both renal pedicles were clamped for 40 minutes simultaneously. As shown in Figure 3C, the degree of BUN elevation was similar on day 1 after IRI $(102 \pm 4 \mathrm{mg} / \mathrm{dl}$ vs. $100 \pm 4 \mathrm{mg} / \mathrm{dl}$ in controls, $n=12-14)$, whereas BUN levels on day 3 after IRI were significantly more elevated in HiflaHif $2 \mathrm{a}^{-/-}$mutants than in controls $(97 \pm 9 \mathrm{mg} / \mathrm{dl}$ vs. $67 \pm 12 \mathrm{mg} / \mathrm{dl}$ respectively, $n=7-6 ; P=0.002)$. Together with findings from the morphologic and molecular analysis of HiflaHif2 $a^{-/-}$mice, the relative increase in BUN levels at day 3 and not at day 1 suggested that endothelial HIF promotes recovery from renal IRI.
To determine whether inactivation of endothelial HIF- $1 \alpha$ and HIF-2 $\alpha$ affected the development of IRI-associated fibrosis, mice were subjected to unilateral renal artery clamping and analyzed 9 days following reperfusion. EC-specific Hifl aHif $2 \mathrm{a}^{-/-}$mice accumulated more collagen in cortex and medulla, as assessed by Sirius red morphometry (cortex: increase by $53 \%, P=0.006$; medulla: increase by $32 \%, n=4, P=0.02$; Figure $4 \mathrm{~A}$ ), which associated with increased transcript levels of collagen $1 \alpha 1$ (Col1a1), Col18a1, lysyl oxidase-like 2 (Loxl2) and Tgfb1 ( $n=4, P<0.05$; Figure 4B). Since significant capillary rarefaction is frequently observed in kidneys with previous ischemic injury (4), we examined peritubular capillary density using CD31 immunohistochemistry and found no differences between mutants and controls (Figure 4C). Next, we assessed the degree of macrophage accumulation in post-IRI kidneys and found an approximately $30 \%$ increase in the number of $54 / 80$-positive cells that infiltrated Hif1aHif2a $a^{-/-}$kidneys $(220 \pm 23$ cells/hpf vs. $171 \pm 7$ cells/hpf, $n=4, P=0.078$; Figure $4 \mathrm{D}$ ). In summary, our data demonstrate that inactivation of endothelial HIF negatively affected recovery from IRI and worsened fibrosis and inflammation found in the post-IRI kidney.

Endothelial HIF suppresses IRI-associated renal inflammation. Our findings suggested that endothelial HIF protects from ischemic renal 
A

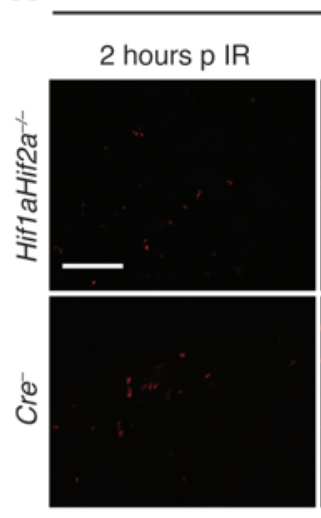

CD45

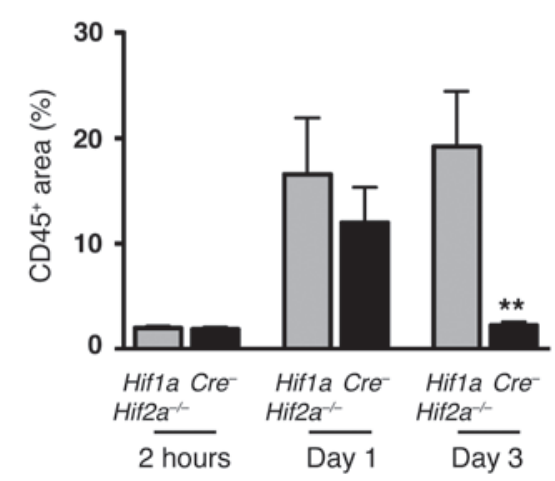

B
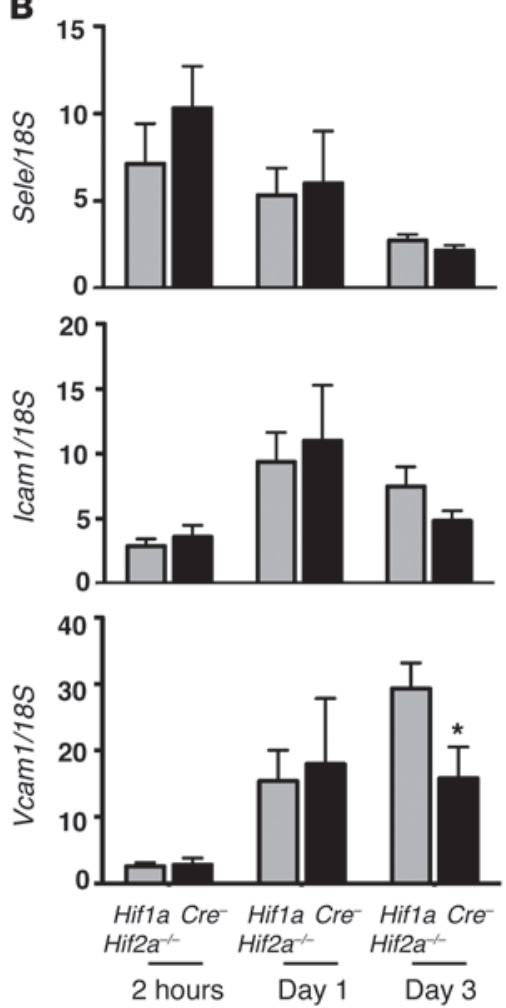

Figure 5

Endothelial HIF suppresses IRI-associated renal inflammation. (A) Representative images of CD45-stained sections of injured kidneys from Hif1 aHif2a $a^{-/}$mutant mice and $\mathrm{Cre}^{-}$littermate controls at 2 hours, 1 day, and 3 days following ischemia reperfusion. Bottom panel shows quantification of CD45-positive area in Hif1aHif2a ${ }^{-/-}$and Cre- controls $(n=6)$. (B) Transcript levels of Sele, Icam1, and Vcam1 in injured kidneys at time points indicated $(n=5-6)$. Graph bars represent mean values \pm SEM. ${ }^{\star} P<0.05 ;{ }^{* \star} P<0.01$. Scale bars: $100 \mu \mathrm{m}$.

injury by modulating inflammatory responses, which play a major role in the pathophysiology of IRI. To gain mechanistic insights, we characterized the time course of leukocyte infiltration by quantitative assessment of CD45 staining in injured kidneys. We found that CD45 expression in HiflaHif2 $a^{-/-}$mutants correlated with the degree of Kim1 upregulation (Figure 5A). While no significant differences were found at the 2-hour and 1-day time points, CD45 staining was increased by 8.6 -fold in mutants at day 3 after IRI $(19.2 \% \pm 5.3 \%$ vs. $2.2 \% \pm 3.3 \%$ in controls, $n=6, P=0.002$; Figure $5 \mathrm{~A}$ ). Time-course analysis of neutrophil infiltration furthermore revealed that the severity of inflammation at days 1 and 3 after IRI remained unchanged in HiflaHif $2 a^{-/-}$kidneys, while neutrophil numbers in $\mathrm{Cre}^{-}$mice were decreased by day 3 , suggesting that the resolution of IRI-associated inflammation was significantly impaired in HiflaHif $2 a^{-/}$mice.

As HIF has been shown to modulate EC barrier function $(20,23)$, we tested whether alterations in renal vascular permeability contributed to increased leukocyte content in Hif1 aHif2 $\mathrm{a}^{-/-}$IRI kidneys. For this purpose, we performed time-course analysis of EBD permeability and found no significant differences between HiflaHif2 $\mathrm{a}^{-/}$ kidneys and controls on days 1, 2, and 3 after IRI (Supplemental Figure 4). This finding suggested that altered vascular permeability is unlikely to have contributed to increased neutrophil accumulation in HiflaHif2 $a^{-/-}$kidneys.

Because ECs regulate inflammation in injured tissues through modulation of neutrophil adhesion, we next examined mRNA expression levels of endothelial adhesion molecules by real-time
PCR. Transcripts levels for E-selectin (Sele), which specifically regulates leukocyte rolling on ECs, were maximally induced 2 hours after IRI and did not differ between mutant and control mice at all time points (Figure 5B). Similarly, significant differences were not found for intercellular adhesion molecule-1 (Icam1), a key regulator of leukocyte adhesion and transendothelial migration (Figure 5B). In contrast, mRNA levels for vascular cell adhesion molecule-1 (Vcam1) were significantly increased in HiflaHif $2 a^{-/-}$ kidneys on day 3 after IRI (a 1.9-fold difference compared with controls, $n=5-6, P<0.05$; Figure 5B).

To investigate to what degree increased Vcam 1 expression contributed to the exacerbation of renal injury in endothelial $\mathrm{HiflaHif2a^{-/ }}$ mutants, we administered inhibitory monoclonal antibodies directed against VCAM1 and its ligand very late antigen-4 (VLA4). Specifically, HiflaHif $2 a^{-/}$and $\mathrm{Cre}^{-}$mice were treated with antiVCAM1/VLA4 antibodies or unspecific control IgG at the time of renal clamping and at day 2 after IRI (Figure 6). Renal injury was assessed on day 3 after IRI by morphological evaluation and analysis of Kim1 mRNA expression levels. We found that kidney injury was ameliorated in HiflaHif2 $a^{-/-}$mice in response to VCAM1 blockade, while treatment of $\mathrm{Cre}^{-}$mice did not result in differences compared with IgG-treated controls (Figure 6). Furthermore, renal myeloperoxidase (MPO) activity, a marker of neutrophil accumulation, was reduced 2.7-fold in HiflaHif2 $\mathrm{a}^{-/-}$mutants treated with anti-VCAM1/VLA4 antibodies compared with controls $(P<0.05)$ (Figure 6A). In line with decreased inflammation were decreased 
A



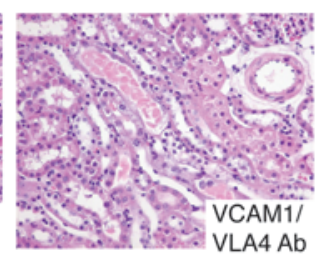
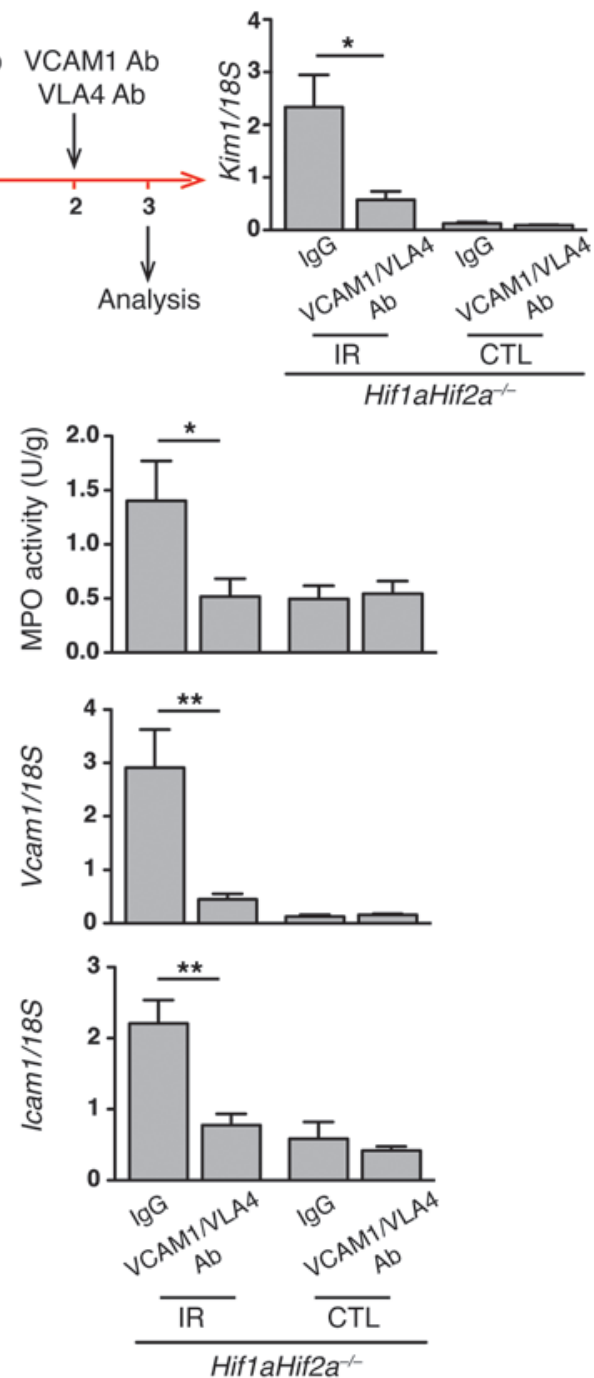

B
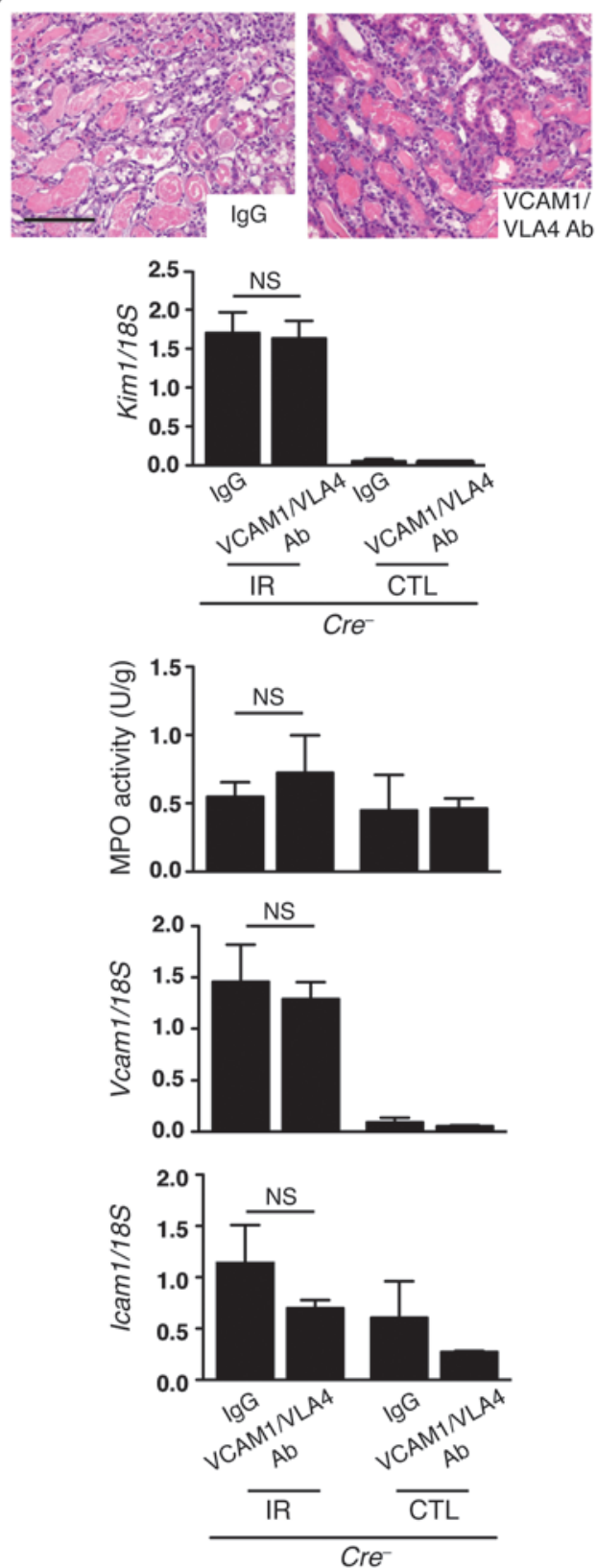

Figure 6

Blockade of VCAM1/VLA4 reverses renal injury associated with endothelial HIF deletion. Overview of the experimental protocol and representative images of H\&E-stained kidney sections from control IgG- or anti-VCAM1/VLA4-treated Hif1aHif2a-/- mutants (A) or Cre- mice (B) on day 3 after IRI. Respective bottom panels show corresponding Kim1 mRNA levels ( $n=6-7)$, MPO activity, and quantitative RT-PCR analysis for Vcam1 and Icam 1 in IR and CTL kidneys on day 3 after IRI. Graph bars represent mean values \pm SEM. ${ }^{*} P<0.05 ;{ }^{*} P<0.01$. Scale bars: $100 \mu \mathrm{m}$.

transcript levels of Vcam1 and Icam1 (Figure 6A). In summary, our data demonstrate that persistent inflammation in HiflaHif2 $a^{-1-}$ IRI kidneys resulted from the increased expression of Vcam1 and suggest that endothelial HIF may suppress IRI-associated inflammation through modulation of EC-neutrophil interaction during the recovery phase from injury.

Inactivation of endothelial HIF-2 $\alpha$ but not HIF-1 $\alpha$ impairs recovery from IRI-associated inflammation. To dissect the contribution of individual HIF homologs to IRI-associated inflammation, we used the Cdh5-Cre transgene to generate mice that were deficient for either HIF- $1 \alpha$ or HIF-2 $\alpha$, from herein referred to as $\mathrm{Hifl}_{1 \mathrm{a}^{-/}}$or Hif $2 \mathrm{a}^{-/-}$mutants. EC-specific $H i f 1 a^{-/-}$or $H i f 2 a^{-/-}$mutants were subjected to unilateral renal artery clamping for 25 minutes and analyzed on day 3 after IRI. CD45-positive cell infiltration increased by 3 -fold in post-IRI $\mathrm{Hif} 2 \mathrm{a}^{-/-}$kidneys compared with $\mathrm{Cr} \mathrm{e}^{-}$littermates, whereas significant differences were not detected between injured $\mathrm{Hifla}^{-/-}$and $\mathrm{Cre}^{-}$kidneys (Figure 7A). Similarly to HiflaHif2a-/- kidneys, CD45-positive cell infiltration in Hif2 $\mathrm{a}^{-/-}$kidneys correlated with Vcam 1 mRNA 
A

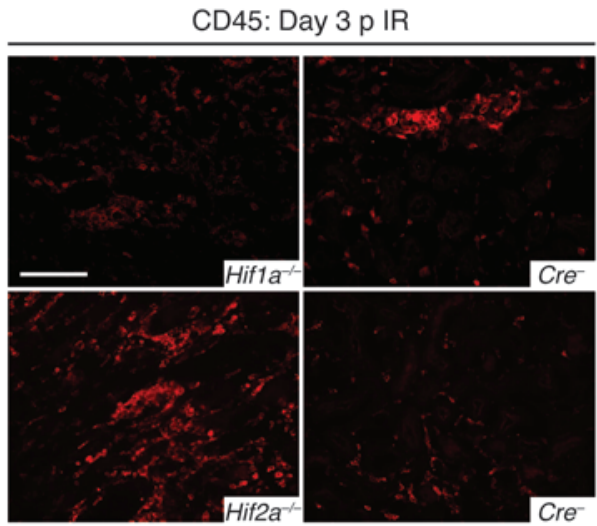

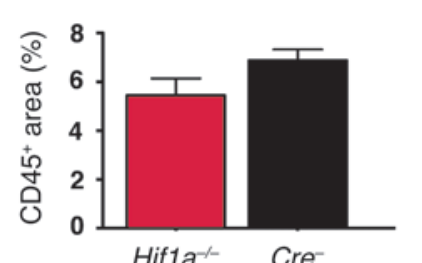

$\mathrm{Hif1}^{-/} \quad \mathrm{Cre}^{-}$

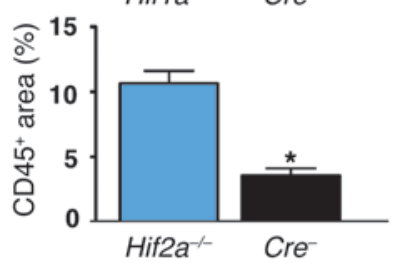

B

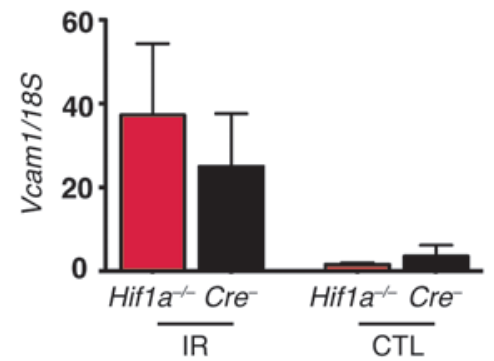

C

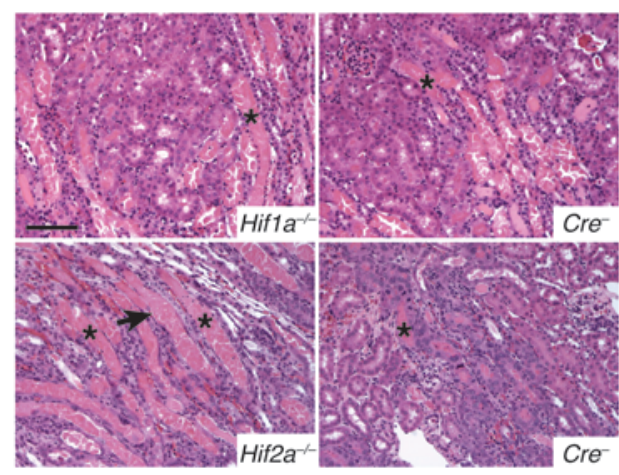

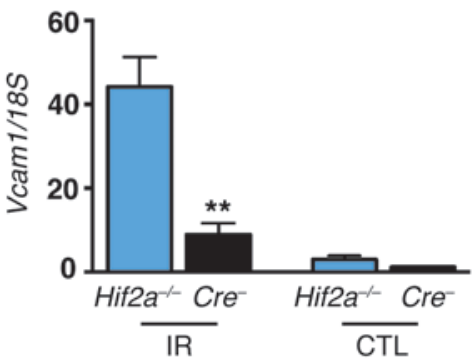
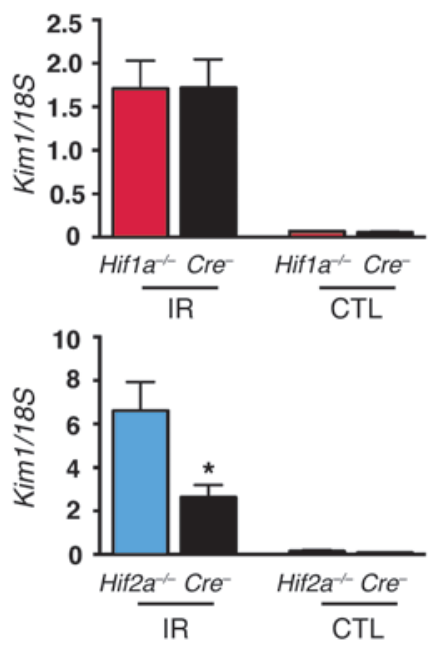

Figure 7

Inactivation of endothelial HIF-2 $\alpha$ but not HIF- $1 \alpha$ impairs recovery from kidney injury and inflammation. (A) Representative images of CD45-stained sections of injured kidneys from EC-specific Hif1 $\mathrm{a}^{-/-}$, Hif2a $\mathrm{a}^{-/}$, or Cre- littermate control mice. Right panel shows percentage of CD45-positive area $(n=3-6)$. (B) Relative Vcam1 mRNA levels in IR and CTL kidneys on day 3 after IRI $(n=5-6)$. (C) Representative images of H\&E-stained kidney sections 3 days after renal IRI in $\mathrm{Hif1}^{-/-}$, Hif2a ${ }^{-1-}$, or Cre- littermate controls. Arrow indicates a necrotic dilated tubule; asterisks mark tubules with cast-forming material. Kim1 mRNA levels in IR and CTL kidneys are shown on right $(n=6)$. Graph bars represent mean values \pm SEM. ${ }^{*} P<0.05$; ${ }^{\star *} P<0.01$. Scale bars: $100 \mu \mathrm{m}$.

expression $(n=5$; Figure 7B), which was upregulated by approximately 5-fold compared with controls, while Vcam1 levels in Hifla $\mathrm{a}^{-1}$ kidneys did not differ from those of controls ( $n=5-6$; Figure $7 \mathrm{~B}$ ). Increased inflammation in Hif2 $\mathrm{a}^{-/-}$mutants was associated with increased injury based on morphologic criteria (Supplemental Figure 5) and on Kim1 expression levels (2.5-fold upregulation, $n=6$, $P=0.02$; Figure 7C). In contrast, histological damage and Kim1
mRNA levels in Hif1 $\mathrm{a}^{-/-}$kidneys were not different from those of controls (Supplemental Figure 5 and Figure 7C), indicating that endothelial HIF-1 and HIF-2 play distinct roles in the development of inflammation and injury associated with renal IRI.

Expression of Cre recombinase under control of the Cdh5 promoter has been demonstrated in hematopoietic cells (14). Although recombination analysis in our model indicated limited activity of Cdh5-Cre in the bone marrow ( $35 \%$ of bone marrow cells, data not shown), we performed bone marrow transplantation experiments to assess for a potential contribution of hematopoietic cells to the exacerbation of renal injury in Hif $2 \mathrm{a}^{-/-}$mutants. For this we transplanted bone marrow cells derived from Hif $2 \mathrm{a}^{-/-}$mutants or WT mice into lethally irradiated WT mice. We predicted that a significant contribution of $\mathrm{Hif} 2 \mathrm{a}^{-/-}$bone marrow-derived cells would exacerbate renal injury in a WT background. However, transplantation of $H i f 2 a^{-/}$bone marrow into WT mice failed to worsen the degree of renal injury and inflammation compared with WT bone marrow (Supplemental Figure 6). We therefore concluded that Cdh5-Cremediated HIF-2 inactivation in hematopoietic cells alone did not affect the outcome of IRI in our model. Taken together, our combined data demonstrate that endothelial HIF-2 plays a specific and critical role in the development of renal injury and inflammation associated with IRI.

Inactivation of endothelial PHD2 attenuates renal IRI and suppresses VCAM1-mediated inflammatory cell adhesion. Based on our findings that inactivation of endothelial HIF exacerbates postischemic renal injury and inflammation, we investigated whether EC-specific activation of HIF would be sufficient to induce renoprotection. To address this question, we crossed Cdh5-Cre transgenic mice with mice homozygous for a floxed $P h d 2$ allele (24), and generated Cdh5Cre Phd2flox/flox mice, herein referred to as $\mathrm{Phd}^{-/-}$mutants. Because PHD2 is the main HIF prolyl-hydroxylase that targets HIF for degradation under normoxia, we predicted that EC-specific loss of $\mathrm{Phd} 2$ would result in HIF stabilization, which we validated by immunoblot and RT-PCR analysis of HIF target genes (data not shown). Remarkably, $\mathrm{Phd2^{-/- }}$ mutants displayed attenuated kidney injury on day 3 after IRI compared with $\mathrm{Cre}^{-}$littermates, as evidenced by substantial improvements in histological injury scores and Kim1 transcript levels (Figure 8A). Furthermore, injured $\mathrm{Phd2^{-/- }}$ kidneys were characterized by approximately 3.5- and 2.4-fold reduction in Vcam1 and Icam1 mRNA levels, respectively, compared with controls (Figure 8B). 
A
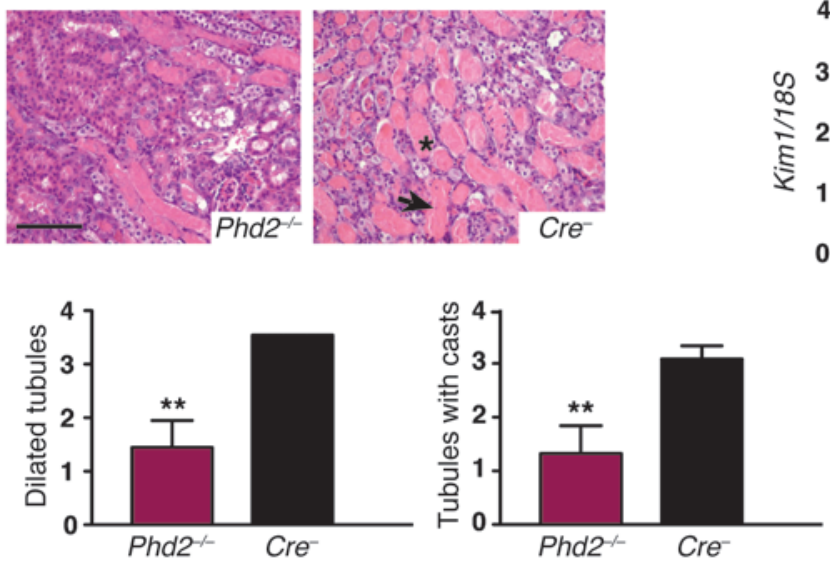

B

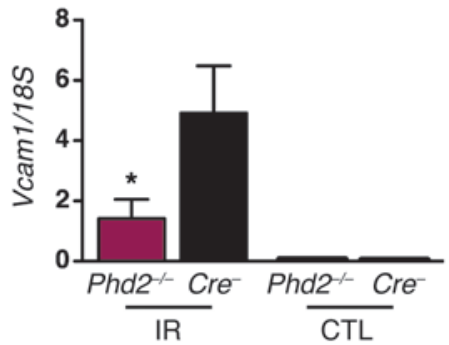

C
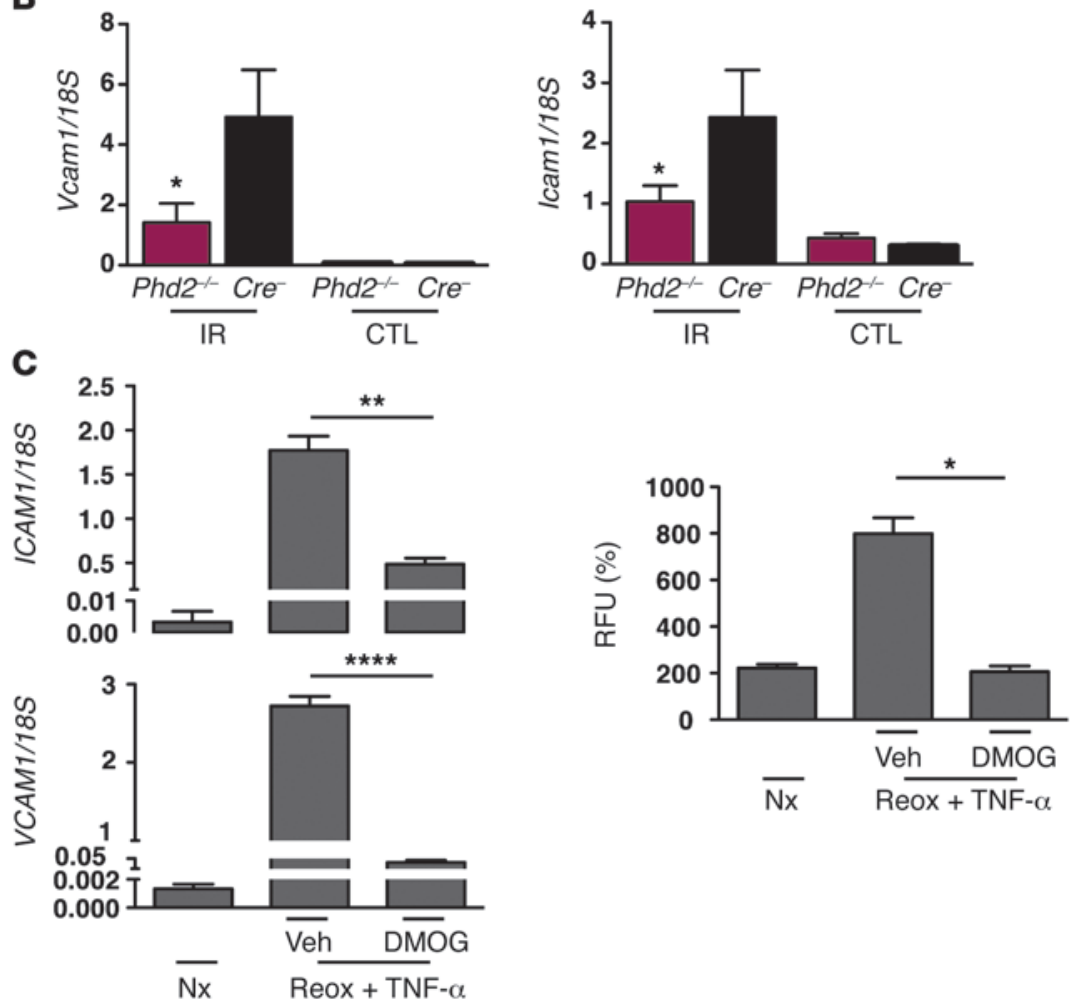

\section{Figure 8}

Inactivation of endothelial PHD2 attenuates renal IRI and suppresses the induction of EC-adhesion molecules. (A) Representative images of $\mathrm{H} \& \mathrm{E}$-stained kidney sections from $\mathrm{Phd}^{-/-}$or $\mathrm{Cre}^{-}$littermate controls 3 days after renal IRI. Arrow depicts tubular necrosis. Asterisk indicates cast. Lower graphs show semiquantitative scores for dilated tubules and tubules with cast-forming material $(n=5-7)$. Kim1 mRNA levels in IR and CTL kidneys are shown on right $(n=5-7)$. (B) Relative Vcam1 and Icam1 mRNA levels in IR and CTL kidneys on day 3 after IRI $(n=5-7)$. (C) HUVECs were treated with DMOG or vehicle during hypoxia exposure and subjected to reoxygenation in the presence of TNF- $\alpha$. Left panels: VCAM1 and ICAM1 mRNA levels in HUVECs. Right panel: functional adhesion assay with fluorochrome-labeled THP-1 cells. THP-1 cells were cocultured for 1 hour with HUVECs; the degree of adhesion is measured in relative fluorescence units. Graph bars represent mean values \pm SEM. ${ }^{*} P<0.05$; ${ }^{*} P<0.01$; ${ }^{* * *} P<0.0001$. Scale bars: $100 \mu \mathrm{m}$. veh, vehicle (DMSO).
To assess whether the effects of endothelial PHD inactivation on Vcam 1 expression were cell autonomous, we conducted hypoxiareoxygenation experiments in vitro with HUVECs in conjunction with pharmacologic HIF prolyl-hydroxylase inhibition. For this we used dimethyloxalylglycine (DMOG), a frequently used cellpermeable pan-HIF prolyl-hydroxylase inhibitor that is capable of effectively stabilizing HIF- $\alpha$ in vitro and in vivo (25). To define the effects of HIF prolyl-hydroxylase inhibition on hypoxiareoxygenation-induced expression of EC-adhesion molecules, HUVECs were incubated for 18 hours at $0.5 \% \mathrm{O}_{2}$ in the absence or presence of $1 \mathrm{mM}$ DMOG followed by reoxygenation for 6 hours in the presence of $1 \mathrm{ng} / \mathrm{ml} \mathrm{TNF-} \alpha$ (Supplemental Figure 7). Hypoxiareoxygenation resulted in an 8 - and 23 -fold increase of ICAM1 and VCAM1 transcript levels, respectively, whereas treatment with DMOG completely abolished the effects of hypoxia-reoxygenation on EC adhesion molecule expression (Figure 8C). Similarly, pre- treatment with DMOG blunted hypoxia-reoxygenation-induced adhesion of monocytes (THP-1 cells) to HUVECs (Figure 8C). Taken together, our studies suggest that inhibition of HIF prolylhydroxylation in ECs is sufficient to induce renoprotection and to suppress inflammatory responses.

Endothelial HIF-2 is required for renoprotection induced by systemic HIF prolyl-hydroxylase inhibition. We next sought to determine whether pharmacologic activation of endothelial HIF-2 could be used to protect from renal injury and inflammation. We previously reported that GSK1002083A, herein referred to as PHI, a structural 2OG analog, stabilizes both HIF- $1 \alpha$ and HIF- $2 \alpha$, induces HIF-regulated genes in the kidney, and protects from renal IRI $(8,12)$. We administered 3 doses of PHI to WT mice subjected to 25 minutes of unilateral renal artery clamping (for dosing regimen, see Figure 9A). PHI treatment attenuated renal injury at day 3 after IRI compared with vehicle-treated controls based on morphologic injury score 
A
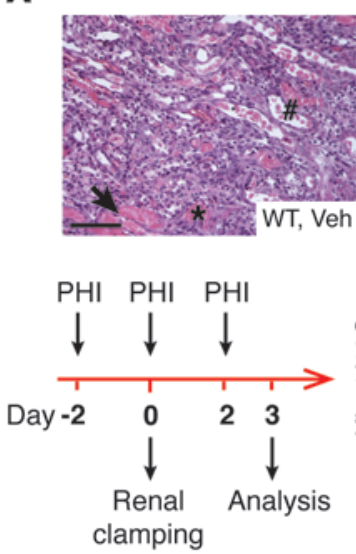

B
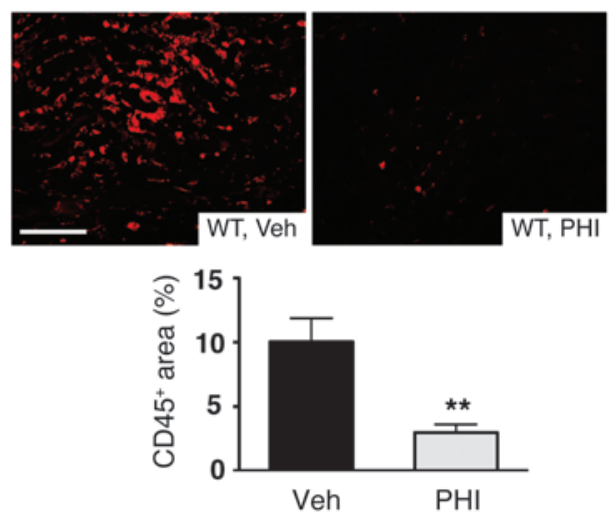

C
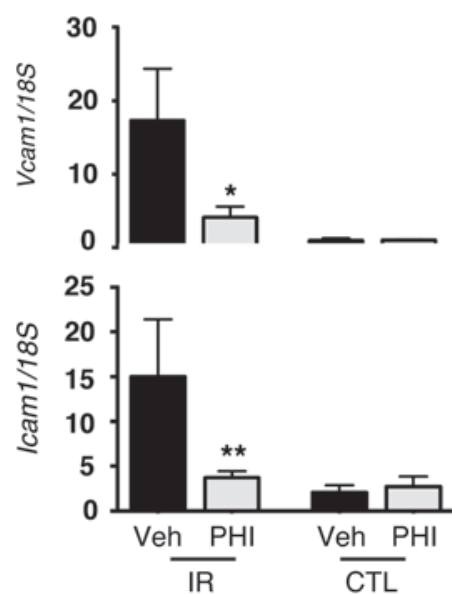

D
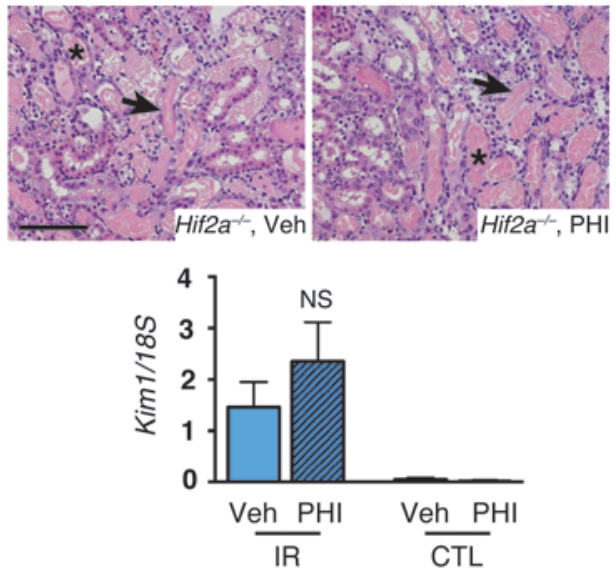

E
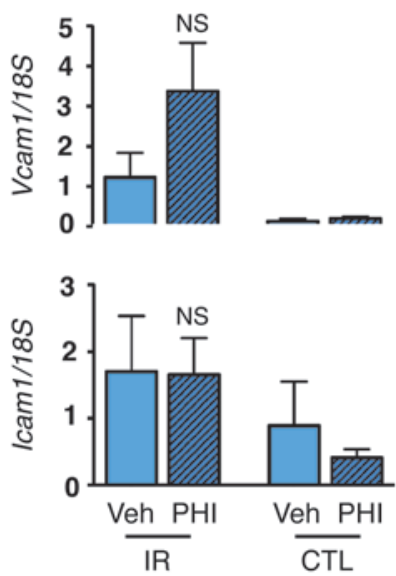

F

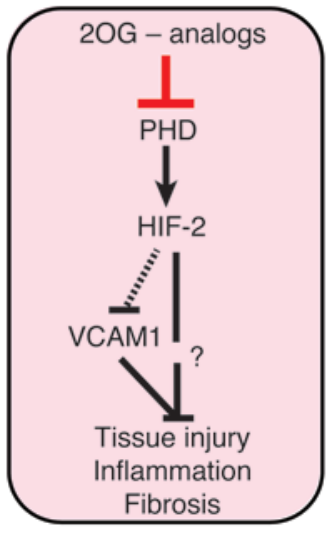

Figure 9

Renoprotection induced by systemic HIF prolyl-hydroxylase inhibition requires endothelial HIF-2. (A) Scheme of the experimental protocol and representative images of H\&E-stained kidney sections from vehicle- or PHI-treated WT animals on day 3 after IRI. Arrow indicates tubular necrosis. Hatch mark shows dilatation. Asterisk indicates casts. Bottom panel on the right shows corresponding Kim1 mRNA levels $(n=6)$. (B) Representative images of CD45-stained sections from injured kidneys of vehicle- or PHI-treated WT animals. Graph shows quantitative analysis of CD45positive area $(n=6)$. (C) Relative levels of Vcam1 and Icam1 mRNA $(n=6)$. (D) Representative images of H\&E-stained kidney sections from $\mathrm{PHI}$ - or vehicle-treated EC-specific Hif2a ${ }^{-/}$mutants and corresponding Kim1 mRNA levels $(n=4-5)$. Arrows depict tubular necrosis. Asterisks indicate casts. (E) Renal transcript levels of Vcam1 and Icam1 in PHI- or vehicle-treated Hif2a $\mathrm{a}^{-/-}$mice $(n=4-5)$. (F) Schematic depicting the role of the endothelial PHD/HIF-2 axis in renal ischemic injury. Graph bars represent mean values \pm SEM. ${ }^{\star} P<0.05 ;{ }^{\star \star} P<0.01$. Scale bars: $100 \mu \mathrm{m}$. PHI, HIF prolyl-hydroxylase inhibitor; veh, vehicle (1\% methylcellulose). 
and $\operatorname{Kim} 1$ expression $(n=6, P<0.05$; Figure 9A and Supplemental Figure 8). Renoprotection was furthermore associated with a reduction in CD45-positive leukocyte content (3-fold reduction compared with controls $n=6, P<0.01$; Figure 9B) and reduced expression of Vcam 1 and Icam 1 transcripts (4.2- and 4.1-fold, $P=0.04$ and $P=0.004$ respectively; $n=6$; Figure 9C), while we did not observe differences in Sele mRNA expression. We next investigated the contribution of endothelial HIF- $2 \alpha$ to renoprotection induced by systemic PHI administration. Since endothelial HIF-2 regulated IRI-associated inflammation, we hypothesized that PHIinduced protection was at least in part due to endothelial HIF-2 activation. To test this hypothesis, Hif $2 \mathrm{a}^{-/-}$mice were treated with $\mathrm{PHI}$ or vehicle alone, subjected to unilateral renal IRI, and analyzed on day 3 after IRI. In contrast with WT mice, PHI treatment did not induce protection in Hif $2 \mathrm{a}^{-/-}$mice when compared with treatment with vehicle alone. Morphologic injury scores and Kim-1, Vcam1, and Icam 1 mRNA levels were not different from those of vehicle-only-treated Hif $2 \mathrm{a}^{-/-}$mice, indicating that endothelial HIF- $2 \alpha$ is required for PHI-induced renoprotection (Figure 9, D and E, and Supplemental Figure 8). Taken together, our studies identified endothelial HIF- $\alpha$ as a critical pharmacologic target that mediates the antiinflammatory and renoprotective effects of HIF-prolyl hydroxylase inhibition (Figure 9F).

\section{Discussion}

In the present study, we used a genetic approach to dissect the role of endothelial HIF-1 and HIF-2 under conditions of hypoxic and/or ischemic renal injury. Utilizing different kidney injury models and conditional knockout strains in which both HIF homologs were activated or inactivated either simultaneously or individually, we provide experimental evidence that endothelial HIF-2, but not HIF-1, regulates renal inflammation, likely through suppression of VCAM1. We furthermore identify endothelial HIF-2 as a therapeutic target that, when activated pharmacologically, has the potential to protect kidneys from the long-term sequelae of acute kidney injury by suppressing inflammation and by promoting tissue repair.

Mice with EC-specific inactivation of both HIF- $1 \alpha$ and HIF- $2 \alpha$ using Cdh5-Cre developed normally, were fertile, and did not display major vascular abnormalities. This was a surprising finding, as the absence of a significant vascular phenotype in EC-specific conditional knockout mice that lacked either HIF- $1 \alpha$ or HIF- $2 \alpha$ was attributed to functional redundancy between HIF-1 and HIF-2 $(20,26,27)$. In contrast, Tie2-Cre-mediated inactivation of the aryl-hydrocarbon receptor nuclear translocator (ARNT), which functions as the HIF- $\beta$ subunit, led to the development of abnormal hepatic vasculature, liver necrosis, degenerative lesions in cardiac myocytes, and neonatal lethality (28). In addition to heterodimerization with HIF- $\alpha$, ARNT is an obligate binding partner of the aryl-hydrocarbon (dioxin) receptor (AHR) and is involved in executing xenobiotic stress responses (29). It is therefore likely that the vascular phenotype in $\mathrm{Arnt}^{-/-}$mice was not due to loss of HIF function, but rather to abrogated AHR signaling. Alternatively, the differences between our Cdh5-Cre-based model and the previously reported Tie2-Cre-based Arnt $t^{\text {flox/flox }}$ model might be related to spatio-temporal differences in Crerecombinase activity, as mice with germ line inactivation of either HIF- $1 \alpha$ or HIF- $2 \alpha$ or mice that expressed a dominant-negative form of endothelial HIF died in utero from cardiovascular malformations $(15,30-32)$.
Our data support a critical role for endothelial HIF-2 $\alpha$, but not HIF-1 $\alpha$, in the regulation of IRI-associated inflammation and recovery from kidney injury. This finding was surprising as, based on functional and genome-wide transcriptional analyses, it is clear that both HIFs mediate endothelial hypoxia responses (20,33-35). Furthermore, endothelial HIF-1 and HIF-2 are both involved in the regulation of tumor angiogenesis and wound healing $(20,26$, $35,36)$. In our study, we found that inactivation of endothelial HIF- $2 \alpha$ impaired the resolution of IRI-associated renal inflammation, which worsened fibrosis and macrophage infiltration during the later phases of renal recovery. This finding is consistent with observations from experimental and clinical studies of transplanted kidneys, in which the degree of IRI-associated inflammation predicted posttransplant graft function and macrophage infiltration at 1 week and 6 months, respectively $(37,38)$.

Resolution of inflammation and recovery from IRI was delayed in Hif $2 a^{-/-}$mutants and was associated with elevated Vcam 1 expression. Remarkably, antibody blockade of VCAM1/VLA4 was sufficient to reverse the effects of EC-specific HIF deletion, indicating that the endothelial HIF-2/VCAM1 axis played a major role in the exacerbation of renal injury associated with endothelial HIF-2 deletion. Since EC barrier function was not affected in postischemic Hif2 $a^{-/-}$kidneys, it is likely that dysregulated Vcam1 expression resulted in prolonged leukocyte adhesion and transendothelial migration, thus worsening inflammation in our model. This hypothesis is supported by several studies that investigated VCAM1's role in murine models of contact dermatitis, granulomatous inflammation, and atherosclerosis and by studies in human kidney transplant biopsies (39-41). An association of Vcam1 expression levels and delayed recovery from renal injury and inflammation has also been found in rats subjected to IRI (42). While blockade of VCAM1/VLA4 ameliorated injury in $\mathrm{Hif}_{2} \mathrm{a}^{-/-}$mutants, it had no impact on renal outcome in WT mice. Similarly, in an ovine model of renal transplantation, blockade of VCAM1-ligand interaction via administration of anti-VCAM1 monoclonal antibodies did not alter allograft survival or mononuclear inflammation (43). Whether a certain VCAM1 expression level is required for VCAM1/ VLA4 blockade to be effective is not clear. Genetic studies are needed to address the role of VCAM1 in renal IRI more conclusively. Nevertheless, our data indicate that the PHD/HIF-2/VCAM1 axis may represent an effective therapeutic target for the treatment and prevention of ischemic renal injury.

Systemic HIF activation by pharmacologic means protects from IRI in several tissues, including experimental models of acute renal failure (10-12). In contrast, animal models of CKD support both renoprotective and injury-promoting roles (21, 44-47). A major challenge has been the identification of cell types and molecular targets that mediate HIF-induced renoprotection. Interestingly, a global knockdown of HIF- $2 \alpha$ in mice resulted in increased susceptibility to renal IRI, which was reversed by restoration of HIF-2 expression in ECs specifically, implicating endothelial HIF-2 in renal cytoprotection. It was hypothesized that renoprotection occurred through its ability to induce ROS-scavenging enzymes (48). While this study uses a different genetic approach in a background of global HIF-2 suppression and focused on the role of HIF-2 signaling on oxidative stress in the immediate post-injury phase ( 24 hours after reperfusion), our data identify a critical function for endothelial HIF- $2 \alpha$ in later phases of kidney injury via regulation of inflammatory cell adhesion. Furthermore, our studies use genetic and pharmacologic means to demonstrate that 
endothelial HIF-2 is required for the generation of renoprotective responses induced by systemic HIF prolyl-hydroxylase inhibition. In this context, endothelial HIF activation may be particularly useful for the prevention or amelioration of long-term sequelae associated with acute kidney injury, such as renal fibrosis and progression to ESRD, which presents a major clinical problem that had not been fully appreciated until recently (2).

While our studies demonstrate that endothelial HIF-2 has a critical role in renoprotection and suppresses IRI-associated inflammation through the modulation of endothelial Vcam1 expression and inflammatory cell adhesion, genetic activation of HIF signaling in other cell types, such as epithelial cells, has been shown to induce cytoprotection in the kidney $(49,50)$. While these responses are complex and not well understood, the molecular mechanism underlying renoprotection may include increased expression of certain cytoprotective factors, oxidative stress responses, and reprogramming of glucose, energy, and adenosine metabolism as well as beneficial effects on mitochondrial $\mathrm{O}_{2}$ utilization and ROS production $(9,45,48,51,52)$. Under certain conditions, these protective effects may be offset by injury-promoting effects ascribed to HIF activation; e.g., chronic HIF activation in tubular epithelial cells has been shown to promote fibrosis via the induction of ECMmodifying and lysyl oxidase genes (21). In the context of pharmacologic HIF prolyl-hydroxylase inhibition, HIF-independent functions of PHDs may contribute to cytoprotective responses (53). For instance, PHDs might function as negative regulators of NF- $\kappa \mathrm{B}$, leading to activation of NF- $\mathrm{KB}$ signaling and modulation of apoptotic responses as a result of HIF prolyl hydroxylase inhibition (53). A question that has yet to be addressed in detail concerns timing of HIF activation for the induction of renoprotection. Recent studies utilizing a postischemic and remnant kidney injury model suggested that timing is critical for eliciting a protective response $(12,13)$.

Our data lend strong support to the notion that HIF-1 and HIF-2 have very distinct and cell type-dependent functions in renoprotection. They furthermore support a concept that ECs, as has been shown for the heart, are critical cellular mediators of ischemic/hypoxic preconditioning in the kidney (54). While endothelial HIF-2 is required for PHI-induced renoprotection, HIF-1 appears to mediate hypoxia tolerance in cardiac myocytes (55). In ischemic myofibers, however, HIF-2 has been shown to be downstream of PHD1-dependent induction of hypoxia tolerance (56). While our in vitro studies are consistent with findings that hypoxic preconditioning suppresses neutrophil adhesion (57), treatment of monocytes with hypoxia or DMOG has been shown to stimulate cell adhesion $(58,59)$. The effects of PHI treatment on Vcam1 expression in IRI kidneys, however, are consistent with observations in human dermal ECs, in which VCAM1 induction by TNF- $\alpha$ was blunted by DMOG in a HIF-dependent manner (60). HIF-dependent suppression of VCAM1 most likely occurs indirectly and could potentially involve increased expression of a transcriptional repressor or decreased expression of certain other transcriptional activators $(61,62)$. Interestingly, PHI treatment in our model also resulted in HIF-2-dependent suppression of Icam1, while only Vcam 1 expression was dysregulated in $\mathrm{Hif} 2 \mathrm{a}^{-/-}$mutant kidneys. To better understand these differences, additional studies are needed that investigate the PHD/HIF axis in the regulation of EC-inflammatory cell interactions in greater detail.

In summary, we have shown by genetic means that endothelial HIF-2 plays a critical role in the regulation of inflammation associated with ischemic kidney injury. We demonstrate that endothelial HIF-2 mediates, at least in part, the antiinflammatory and renoprotective effects of pharmacologic preconditioning induced by PHD inhibitors. Our data therefore provide new insights into the cell type-specific roles of HIF transcription factors in renal disease and identify endothelial HIF-2 as a potential target for renoprotective therapies.

\section{Methods}

Generation of mice and genotyping. The generation and genotyping of Hif1a, Hif2a (also known as Epas1), and $P b d 2$ conditional alleles have been described previously $(15,16,24)$. For the inactivation or activation of HIF- $1 \alpha$ and HIF- $2 \alpha$ in ECs, Cdh5-Cre transgenic mice (14) were crossed to mice carrying conditional Hifla and/or Hif2a or Pbd2 alleles. The following genotypes were generated: (a) Cdb5-Cre Hif1 $a^{f l o x / f l o x} \mathrm{Hif2} a^{\text {flox/flox }}$, referred to as HiflaHif2a-/mutants, (b) Cdh5-Cre Hif1 allox/flox, referred to as Hif1 $a^{-/}$, (c) Cdh5-Cre

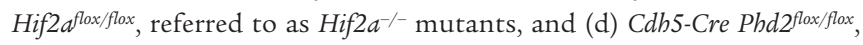
referred to as $P h d 2^{-/-}$mutants. Mice were generated in a mixed genetic background (BALB/c, 129Sv/J, and C57BL/6). For PHI and bone marrow transplantation experiments, WT and Hif2- $\mathrm{a}^{-/}$mutants in a C57BL/6 background were used. Littermates not carrying the Cre transgene served as control animals in all experiments. To assess recombination efficiency, $\mathrm{C} d b 5$-Cre transgenic mice were crossed to ROSA26-ACTB-tdTomato,-EGFP reporter mice (The Jackson Laboratory, strain 007576; ref. 17).

Kidney injury models. Unilateral or bilateral IRI was induced in male mice by clamping the renal pedicle for 25 minutes (unilateral IRI) or for 40 minutes (bilateral IRI) as previously described (12). For pharmacologic HIF activation, HIF prolyl-4-hydroxylase inhibitor GSK 1002083A (GlaxoSmithKline) was dissolved in $1 \%$ methylcellulose and administered by oral gavage at a dose of $60 \mathrm{mg} / \mathrm{kg}$. Mice subjected to renal IRI received a total of 3 doses of GSK compound at 2 days and 6 hours prior to IRI and 2 days after IRI. Blockade of VCAM1/VLA4 interaction was performed by i.v. administration of a monoclonal antibody to VCAM1 (clone M/K 2.7; Bio X Cell) in combination with a monoclonal antibody to VLA4 (clone PS/2; Millipore) or nonspecific control IgG purified from rat serum (Sigma-Aldrich). For kidney IRI, mice received antibodies at the time of renal clamping and 2 days after IRI. For UUO, mice underwent ligation of the right ureter just below the renal pelvis through a back incision; contralateral kidneys were used as controls as previously described (21). For morphologic evaluation, EBD assay, gene expression, and protein analyses, kidneys were harvested 2 hours after IRI or on days 1, 3, or 9 after IRI. For UUO experiments, kidneys were harvested on day 8 or 12 after procedure.

Creation of chimeric mice. Bone marrow transplantation was performed as previously described (63). Briefly, recipient mice were lethally irradiated with 9 Gy via a cesium $\gamma$ source 4 hours prior to transplantation. Bone marrow cells were harvested from the femurs and tibias of donor mice in RPMI-1640. Recipient mice were administered approximately $5 \times 10^{6}$ bone marrow cells in $0.2 \mathrm{ml}$ medium via tail-vein injection. Five weeks after transplantation, recipient mice were subjected to unilateral renal IRI. DNA from whole blood was used to determine the degree of chimerism by PCR.

Vascular permeability assay. Anesthetized mice were administered 1\% EBD (Sigma-Aldrich) by retroorbital injection at a dose of $20 \mathrm{mg} / \mathrm{kg}$ followed by intracardial perfusion with $20 \mathrm{ml}$ PBS and EDTA. Kidneys and lungs were removed and allowed to dry overnight at $55^{\circ} \mathrm{C}$; EBD was extracted in formamide ( $20 \mathrm{ml} / \mathrm{g}$ dry tissue; Sigma-Aldrich). Extracted EBD was measured by optical absorbance at $620 \mathrm{~nm}$, and results were expressed as micrograms of EBD per dry weight of tissue in grams.

Blood, tissue, and urine analysis. Complete blood counts were determined with a Hemavet 950 analyzer (Drew Scientific). Urine albumin and creatinine concentrations were measured using the ELISA Albuwell M Test Kit (Exocel). Blood glucose, serum electrolytes and BUN were measured 
with the iSTAT portable clinical analyzer (Abbott Portable Handheld Clinical Analyzers) and the QuantiChrom Urea Assay Kit (BioAssay Systems). Serum Epo levels were determined with a commercially available ELISA kit according to the manufacturer's instructions (R\&D Systems). MPO activity in kidney homogenates was measured using the MPO Colorimetric Activity Assay Kit according to the manufacturer's instructions (Biovision) and was expressed as U/wet tissue weight.

$D N A$ and RNA analysis. RNA was isolated with Trizol reagent (Invitrogen) followed by clean-up with the RNeasy Mini Kit (QIAGEN) according to the manufacturer's instructions. For real-time PCR analysis, RNA was reverse transcribed and subjected to PCR amplification using either SYBR Green PCR Master Mix or TaqMan Universal PCR Master Mix on an ABI 7300 platform (Applied Biosystems). mRNA expression levels were quantified using the relative standard curve method according to the manufacturer's instructions (Applied Biosystems). Mouse primer sequences for Kim1, Col1a1, Col18a1, Loxl2, and Tgfb1 have been described elsewhere $(12,21)$. The following mouse primer pairs were used for amplification: Cd31: forward, 5'-GTCGTCCATGTCCCGAGAA-3', reverse, 5'-GCACAGGACTCTCGCAATCC-3'; Vcam1: forward, 5'-TAGAGTGCAAGGAGTTCGGG-3', reverse, 5'-CCGGCATATACGAGTGTGAA-3'; Icam 1: forward, 5'-TGGATACCTGAGCATCACCA-3', reverse, 5'-CTGCTACCTGCACTTTGCC-3'; and Sele: forward, 5'-AGGCAAGAGGAACCAGGATT-3', reverse, $5^{\prime}$-ACCATGTGCCTTCTTACAACG-3'. Human primer pairs were as follows: ICAM1: forward, 5'-CCACAGTCACCTATGGCAAC-3'; reverse, 5'-AGTGTCTCCTGGCTCTGGTT-3'; SELE: forward, 5'-TGAACCCAACAATAGGCAAA-3'; reverse, 5'-CCTCTCATCATTCCACATGC-3'; VCAM1: forward, 5'-GCTTCAGGAGCTGAATACCC-3', reverse, 5' AAGGATCACGACCATCTTCC-3'. 18S was used for normalization.

Morphologic analysis. For morphologic analysis, kidneys were fixed with $10 \%$ buffered formalin. Immunofluorescence studies were carried out on tissues that were immersion fixed in $100 \%$ ice-cold methanol. Leukocyte and macrophage infiltration was assessed with a monoclonal rat anti-CD45 antibody (eBioscience) and a monoclonal rat anti-F4/80 antibody (Abcam Inc). Capillary density was examined with a polyclonal rabbit anti-cablin antibody (generated against recombinant protein as previously described, ref. 19) and a monoclonal rat anti-CD31 antibody (BD Biosciences). To assess ECM accumulation, we performed Sirius red staining ( $0.1 \%$ fast green FCF and $0.1 \%$ direct red 80 in saturated picric acid). For all morphologic quantifications, 10 random visual fields were analyzed per kidney section, and percentage of Sirius red, CD31- and CD45-positive area, or number of F4/80-positive cells were determined with ImageJ software (http://rsbweb.nih.gov/ij). The degree of renal injury was assessed morphologically by determining the percentage of dilated and cast-filled tubules and was scored semiquantitatively (0, 0\%-5\%; 1, 6\%-10\%; 2, 11\%-25\%; 3, 26\%-45\%; 4, 46\%-75\%; 5, 76\%-100\%). Fluorescence imaging was performed with a Zeiss LSM 510 confocal laserscanning microscope equipped with Ar and HeNe lasers.

FACS analysis. After perfusion with $10 \mathrm{ml} \mathrm{PBS}$, tissues were minced and incubated in digestive solution (serum free, $0.1 \%$ collagenase A, and $100 \mathrm{U} / \mathrm{ml}$ Dnase I). Following addition of $10 \%$ FBS, cell suspensions were filtered through a $100 \mu \mathrm{m}$ and a $40 \mu \mathrm{m}$ nylon mesh, centrifuged $(300 \mathrm{~g}, 5$ minutes), resuspended in rbc lysis buffer, centrifuged, and then suspended again in $1 \times$ PBS. Single-cell suspensions $\left(2 \times 10^{6} \mathrm{cells} / \mathrm{ml}\right)$ were incubated with FcR-blocking reagent and labeled with APC-conjugated anti-mouse CD31 (clone 390; eBioscience) and V450-conjugated anti-CD45 (clone 30-F11;eBioscience) antibodies.

Cell culture. HUVECs were obtained from ATCC and grown on gelatincoated dishes in Vascular Cell Basal Medium (PCS-100-030; ATCC) supplemented with microvascular endothelial growth factors (PCS-100-041; ATCC). During hypoxic incubation, cells were treated with $1 \mathrm{mM} \mathrm{DMOG}$ (Sigma-Aldrich) or vehicle (DMSO). Hypoxic conditions were achieved by exposing cells to $0.5 \% \mathrm{O}_{2}, 5 \% \mathrm{CO}_{2}$ and $95 \%$ humidity in an Invivo 2200 hypoxia chamber (Ruskinn Technologies). During reoxygenation, cells were treated with $1 \mathrm{ng} / \mathrm{ml}$ TNF- $\alpha$ (Cell Biolabs). To determine adhesion of human monocytes (THP-1; ATCC) to HUVECs, the Cytoselect LeukocyteEndothelium Adhesion Assay Kit was used in accordance with the manufacturer's instructions (Cell Biolabs).

Statistics. All data are reported as mean values \pm SEM. Statistical analyses were performed with Prism 5.0b (GraphPad Software) or R, version 2.15.1 (http:www.r-project.org). Univariate analysis was done using Student's $t$ test or the Mann-Whitney $U$ test unless specified otherwise. A linear regression model was used to assess the effect of group on continuous outcome when appropriate. A 2-tailed significance level of 5\% was considered statistically significant.

Study approval. All procedures involving mice were performed in accordance with NIH guidelines for the use and care of live animals and were approved by the Institutional Animal Care and Use Committee (IACUC) of Vanderbilt University.

\section{Acknowledgments}

This work was supported by the Krick-Brooks chair in Nephrology (to V.H. Haase), NIH grants (R01-DK081646 to V.H. Haase; R01-DK007124 to T.A. Sutton), the Vanderbilt University O'Brien Center (P30-DK07934), the Indiana University O'Brien Center (P30-DK079312), an AMGEN Nephrology Fellowship Grant (to P.P. Kapitsinou), and an American Heart Association National Scientist Development grant (11SDG5570023 to P.P. Kapitsinou).

Received for publication January 29, 2013, and accepted in revised form March 13, 2014.

Address correspondence to: Volker H. Haase, Division of Nephrology and Hypertension, Vanderbilt University Medical Center, C-3119A MCN, 1161 21st Avenue So., Nashville, Tennessee 372322372, USA. Phone: 615.343.7254; Fax: 615.322.6854; E-mail: volker. haase@vanderbilt.edu.

Hideto Sano's present address is: Department of Medical Physiology, Hamamatsu University School of Medicine, Hamamatsu, Japan.
1. Aird WC. Phenotypic heterogeneity of the endothelium: I. Structure, function, and mechanisms. Circ Res. 2007;100(2):158-173.

2. Sanoff S, Okusa MD. Impact of acute kidney injury on chronic kidney disease and its progression. Contrib Nephrol. 2011;171:213-217.

3. Uchino S, et al. Acute renal failure in critically ill patients: a multinational, multicenter study. JAMA. 2005;294(7):813-818.

4. Basile DP. The endothelial cell in ischemic acute kidney injury: implications for acute and chronic function. Kidney Int. 2007;72(2):151-156.

5. Sutton TA, Fisher CJ, Molitoris BA. Microvas- cular endothelial injury and dysfunction during ischemic acute renal failure. Kidney Int. 2002; 62(5):1539-1549.

6. Majmundar AJ, Wong WJ, Simon MC. Hypoxiainducible factors and the response to hypoxic stress. Mol Cell. 2010;40(2):294-309.

7. Kaelin WG Jr, Ratcliffe PJ. Oxygen sensing by metazoans: the central role of the HIF hydroxylase pathway. Mol Cell. 2008;30(4):393-402.

8. Kapitsinou PP, et al. Hepatic HIF-2 regulates erythropoietic responses to hypoxia in renal anemia. Blood. 2010;116(16):3039-3048.

9. Haase VH. Hypoxia-inducible factors in the kidney.
Am J Physiol Renal Physiol. 2006;291(2):F271-F281.

10. Bernhardt WM, et al. Preconditional activation of hypoxia-inducible factors ameliorates ischemic acute renal failure. J Am Soc Nephrol. 2006; 17(7):1970-1978.

11. Hill P, et al. Inhibition of hypoxia inducible factor hydroxylases protects against renal ischemia-reperfusion injury. J Am Soc Nephrol. 2008;19(1):39-46.

12. Kapitsinou PP, et al. Pre-ischemic targeting of HIF prolyl hydroxylation inhibits fibrosis associated with acute kidney injury. Am J Physiol Renal Physiol. 2012;302(9):F1172-F1179.

13. Yu X, et al. The balance of beneficial and deleteri- 
ous effects of hypoxia-inducible factor activation by prolyl hydroxylase inhibitor in rat remnant kidney depends on the timing of administration. Nephrol Dial Transplant. 2012;27(8):3110-3119.

14. Alva JA, et al. VE-Cadherin-Cre-recombinase transgenic mouse: a tool for lineage analysis and gene deletion in endothelial cells. Dev Dyn. 2006; 235(3):759-767.

15. Ryan HE, Lo J, Johnson RS. HIF-1 alpha is required for solid tumor formation and embryonic vascularization. EMBO J. 1998;17(11):3005-3015.

16. Gruber M, Hu CJ, Johnson RS, Brown EJ, Keith B, Simon MC. Acute postnatal ablation of Hif- $2 \alpha$ results in anemia. Proc Natl Acad Sci U S A. 2007; 104(7):2301-2306

17. Muzumdar MD, Tasic B, Miyamichi K, Li L, Luo L. A global double-fluorescent Cre reporter mouse. Genesis. 2007;45(9):593-605.

18. Yamashita T, Ohneda O, Sakiyama A, Iwata F, Ohneda K, Fujii-Kuriyama Y. The microenvironment for erythropoiesis is regulated by HIF- $2 \alpha$ through VCAM-1 in endothelial cells. Blood. 2008; 112(4):1482-1492

19. Charron AJ, Xu W, Bacallao RL, Wandinger-Ness A. Cablin: a novel protein of the capillary basal lamina. Am J Physiol. 1999;277(5 pt 2):H1985-H1996.

20. Skuli N, et al. Endothelial deletion of hypoxiainducible factor-2alpha (HIF-2 $\alpha$ ) alters vascular function and tumor angiogenesis. Blood. 2009; 114(2):469-477.

21. Higgins DF, et al. Hypoxia promotes fibrogenesis in vivo via HIF-1 stimulation of epithelial-to-mesenchymal transition. J Clin Invest. 2007;117(12):3810-3820.

22. van Timmeren MM, van den Heuvel MC, Bailly V, Bakker SJ, van Goor H, Stegeman CA. Tubular kidney injury molecule-1 (KIM-1) in human renal disease. J Pathol. 2007;212(2):209-217.

23. Mazzone M, et al. Heterozygous deficiency of PHD2 restores tumor oxygenation and inhibits metastasis via endothelial normalization. Cell. 2009; 136(5):839-851.

24. Minamishima Y, Moslehi J, Bardeesy N, Cullen $\mathrm{D}$, Bronson R, Kaelin W. Somatic inactivation of the PHD2 prolyl hydroxylase causes polycythemia and congestive heart failure. Blood. 2008 ; 111(6):3236-3244.

25. Elvidge GP, Glenny L, Appelhoff RJ, Ratcliffe PJ, Ragoussis J, Gleadle JM. Concordant regulation of gene expression by hypoxia and 2-oxoglutaratedependent dioxygenase inhibition: the role of HIF- $1 \alpha$, HIF- $2 \alpha$, and other pathways. J Biol Chem. 2006;281(22):15215-15226

26. Tang N, et al. Loss of HIF-1alpha in endothelial cells disrupts a hypoxia-driven VEGF autocrine loop necessary for tumorigenesis. Cancer Cell. 2004; 6(5):485-495.

27. Skuli N, Simon MC. HIF- $1 \alpha$ versus HIF- $2 \alpha$ in endothelial cells and vascular functions: is there a master in angiogenesis regulation? Cell Cycle. 2009; 8(20):3252-3253.

28. Yim SH, et al. Disruption of the Arnt gene in endothelial cells causes hepatic vascular defects and partial embryonic lethality in mice. Hepatology. 2006; 44(3):550-560

29. Reyes H, Reisz-Porszasz S, Hankinson O. Identification of the Ah receptor nuclear translocator protein (Arnt) as a component of the DNA binding form of the Ah receptor. Science. 1992; 256(5060):1193-1195.
30. Iyer NV, et al. Cellular and developmental control of $\mathrm{O} 2$ homeostasis by hypoxia- inducible factor $1 \alpha$. Genes Dev. 1998;12(2):149-162.

31. Peng J, Zhang L, Drysdale L, Fong GH. The transcription factor EPAS-1/hypoxia-inducible factor $2 \alpha$ plays an important role in vascular remodeling. Proc Natl Acad Sci U S A. 2000;97(15):8386-8391.

32. Licht AH, Müller-Holtkamp F, Flamme I, Breier G. Inhibition of hypoxia-inducible factor activity in endothelial cells disrupts embryonic cardiovascular development. Blood. 2006;107(2):584-590.

33. Branco-Price C, et al. Endothelial cell HIF-1 $\alpha$ and HIF- $2 \alpha$ differentially regulate metastatic success. Cancer Cell. 2012;21(1):52-65.

34. Manalo DJ, et al. Transcriptional regulation of vascular endothelial cell responses to hypoxia by HIF-1. Blood. 2005;105(2):659-669.

35 . Skuli N, et al. Endothelial HIF- $2 \alpha$ regulates murine pathological angiogenesis and revascularization processes. J Clin Invest. 2012;122(4):1427-1443.

36. Sarkar K, et al. Tie2-dependent knockout of HIF-1 impairs burn wound vascularization and homing of bone marrow-derived angiogenic cells. Cardiovasc Res. 2012;93(1):162-169.

37. Koo DD, Welsh KI, Roake JA, Morris PJ, Fuggle SV. Ischemia/reperfusion injury in human kidney transplantation: an immunohistochemical analysis of changes after reperfusion. Am J Pathol. 1998; 153(2):557-566.

38. Dragun $\mathrm{D}$, et al. Ischemia-reperfusion injury in renal transplantation is independent of the immunologic background. Kidney Int. 2000;58(5):2166-2177.

39. Briscoe DM, Pober JS, Harmon WE, Cotran RS. Expression of vascular cell adhesion molecule-1 in human renal allografts. J Am Soc Nephrol. 1992; 3(5):1180-1185.

40. Henseleit U, Steinbrink K, Sunderkotter C, Goebeler M, Roth J, Sorg C. Expression of murine VCAM-1 in vitro and in different models of inflammation in vivo: correlation with immigration of monocytes. Exp Dermatol. 1994;3(6):249-256.

41. Cybulsky MI, et al. A major role for VCAM-1, but not ICAM-1, in early atherosclerosis. J Clin Invest. 2001; 107(10):1255-1262

42. Sáenz-Morales D, et al. Differential resolution of inflammation and recovery after renal ischemia-reperfusion injury in Brown Norway compared with Sprague Dawley rats. Kidney Int. 2010; 77(9):781-793

43. Grooby WL, Krishnan R, Johnston JK, Rao MM, Russ GR. Combined anti-vascular cell adhesion molecule- 1 and anti-leukocyte function-associated molecule-1 monoclonal antibody therapy does not prolong allograft survival in an ovine model of renal transplantation. Transplantation. 1998; 66(7):920-924.

44. Kimura K, et al. Stable expression of HIF- $1 \alpha$ in tubular epithelial cells promotes interstitial fibrosis. AmJ Physiol Renal Physiol. 2008;295(4):F1023-F1029.

45. Kobayashi $\mathrm{H}$, et al. Myeloid cell-derived hypoxiainducible factor attenuates inflammation in unilateral ureteral obstruction-induced kidney injury. J Immunol. 2012;188(10):5106-5115.

46. Tanaka $\mathrm{T}$, et al. Cobalt promotes angiogenesis via hypoxia-inducible factor and protects tubulointerstitium in the remnant kidney model. Lab Invest. 2005;85(10):1292-1307.

47. Tanaka $\mathrm{T}$, et al. Induction of protective genes by cobalt ameliorates tubulointerstitial injury in the progressive Thy1 nephritis. Kidney Int. 2005; 68(6):2714-2725.

48. Kojima I, et al. Protective role of hypoxia-inducible factor- $2 \alpha$ against ischemic damage and oxidative stress in the kidney. J Am Soc Nephrol. 2007; 18(4):1218-1226.

49. Fähling M, et al. Tubular von Hippel-Lindau knockout protects against rhabdomyolysis-induced AKI. J Am Soc Nephrol. 2013;24(11):1806-1819.

50. Schley G, et al. Hypoxia-inducible transcription factors stabilization in the thick ascending limb protects against ischemic acute kidney injury. $J \mathrm{Am}$ Soc Nephrol. 2011;22(11):2004-2015.

51. Bauerle JD, Grenz A, Kim JH, Lee HT, Eltzschig HK. Adenosine generation and signaling during acute kidney injury. J Am Soc Nephrol. 2011;22(1):14-20.

52. Bernhardt WM, Warnecke C, Willam C, Tanaka T, Wiesener MS, Eckardt KU. Organ protection by hypoxia and hypoxia-inducible factors. Methods Enzymol. 2007;435:221-245.

53. Wong BW, Kuchnio A, Bruning U, Carmeliet P. Emerging novel functions of the oxygen-sensing prolyl hydroxylase domain enzymes. Trends Biochem Sci. 2013;38(1):3-11

54. Laude K, Beauchamp P, Thuillez C, Richard V. Endothelial protective effects of preconditioning. Cardiovasc Res. 2002;55(3):466-473

55. Loor G, Schumacker PT. Role of hypoxia-inducible factor in cell survival during myocardial ischemiareperfusion. Cell Death Differ. 2008;15(4):686-690.

56. Aragones J, et al. Deficiency or inhibition of oxygen sensor Phd1 induces hypoxia tolerance by reprogramming basal metabolism. Nat Genet. 2008; 40(2):170-180.

57. Beauchamp P, et al. Protective effects of preconditioning in cultured rat endothelial cells: effects on neutrophil adhesion and expression of ICAM-1 after anoxia and reoxygenation. Circulation. 1999; 100(5):541-546

58. Winning S, Splettstoesser F, Fandrey J, Frede S. Acute hypoxia induces HIF-independent monocyte adhesion to endothelial cells through increased intercellular adhesion molecule- 1 expression: the role of hypoxic inhibition of prolyl hydroxylase activity for the induction of NF-кB. J Immunol. 2010; 185(3):1786-1793.

59. Kong T, Eltzschig HK, Karhausen J, Colgan SP, Shelley CS. Leukocyte adhesion during hypoxia is mediated by HIF-1-dependent induction of $\beta 2$ integrin gene expression. Proc Natl Acad Sci U S A. 2004; 101(28):10440-10445.

60. Cartee TV, White KJ, Newton-West M, Swerlick RA. Hypoxia and hypoxia mimetics inhibit TNF-dependent VCAM1 induction in the 5A32 endothelial cell line via a hypoxia inducible factor dependent mechanism. J Dermatol Sci. 2012;65(2):86-94.

61. Schödel J, Oikonomopoulos S, Ragoussis J, Pugh CW, Ratcliffe PJ, Mole DR. High-resolution genome-wide mapping of HIF-binding sites by ChIP-seq. Blood. 2011;117(23):e207-e217.

62. Yun Z, Maecker HL, Johnson RS, Giaccia AJ. Inhibition of PPAR $\gamma 2$ gene expression by the HIF-1-regulated gene DEC1/Stra13: a mechanism for regulation of adipogenesis by hypoxia. Dev Cell. 2002; 2(3):331-341.

63. Nishida M, et al. Absence of angiotensin II type 1 receptor in bone marrow-derived cells is detrimental in the evolution of renal fibrosis. J Clin Invest. 2002;110(12):1859-1868. 